\title{
Identifying Bottom-Up and Top-Down Components of Attentional Weight by Experimental Analysis and Computational Modeling
}

Nordfang, Maria; Dyrholm, Mads; Bundesen, Claus

Published in:

Journal of Experimental Psychology: General

DOI:

10.1037/a0029631

Publication date:

2013

Document version

Early version, also known as pre-print

Citation for published version (APA):

Nordfang, M., Dyrholm, M., \& Bundesen, C. (2013). Identifying Bottom-Up and Top-Down Components of Attentional Weight by Experimental Analysis and Computational Modeling. Journal of Experimental Psychology: General, 142(2), 510-537. https://doi.org/10.1037/a0029631 


\section{Journal of Experimental Psychology: General}

\section{Identifying Bottom-Up and Top-Down Components of Attentional Weight by Experimental Analysis and Computational Modeling}

Maria Nordfang, Mads Dyrholm, and Claus Bundesen

Online First Publication, August 13, 2012. doi: 10.1037/a0029631

\section{CITATION}

Nordfang, M., Dyrholm, M., \& Bundesen, C. (2012, August 13). Identifying Bottom-Up and Top-Down Components of Attentional Weight by Experimental Analysis and Computational Modeling. Journal of Experimental Psychology: General. Advance online publication. doi: $10.1037 / a 0029631$ 


\title{
Identifying Bottom-Up and Top-Down Components of Attentional Weight by Experimental Analysis and Computational Modeling
}

\author{
Maria Nordfang, Mads Dyrholm, and Claus Bundesen \\ University of Copenhagen
}

\begin{abstract}
The attentional weight of a visual object depends on the contrast of the features of the object to its local surroundings (feature contrast) and the relevance of the features to one's goals (feature relevance). We investigated the dependency in partial report experiments with briefly presented stimuli but unspeeded responses. The task was to report the letters from a mixture of letters (targets) and digits (distractors). Color was irrelevant to the task, but many stimulus displays contained an item (target or distractor) in a deviant color (a color singleton). The results showed concurrent effects of feature contrast (color singleton vs. nonsingleton) and relevance (target vs. distractor). A singleton target had a higher probability of being reported than did a nonsingleton target, and a singleton distractor interfered more strongly with report of targets than did a nonsingleton distractor. Measured by use of Bundesen's (1990) computational theory of visual attention, the attentional weight of a singleton object was nearly proportional to the weight of an otherwise similar nonsingleton object, with a factor of proportionality that increased with the strength of the feature contrast of the singleton. This result is explained by generalizing the weight equation of Bundesen's (1990) theory of visual attention such that the attentional weight of an object becomes a product of a bottom-up (feature contrast) and a top-down (feature relevance) component.
\end{abstract}

Keywords: visual attention, stimulus-driven, goal-driven, theory of visual attention, computational modeling

Some of the things that meet our eyes are attended and enter our mind; others go unnoticed. In complex natural scenes, a great multitude of different objects might be noticed, and for each of these objects, the likelihood that the object is noticed depends on many different factors. Some factors are relatively objective, in the sense that they depend strongly on the object but little on the subject. For example, at the marketplace, a particular piece of fruit may be noticed because it sticks out from its surroundings by having a deviant color. Such factors can be regarded as bottom-up components of the attentional weights (cf. Bundesen, 1990) of objects. Other factors are more subjective, depending more strongly on the subject. For example, at the marketplace, another piece of fruit may be noticed because it is needed for a meal one has planned to prepare. Such factors can be regarded as top-down components of attentional weights. The two types of factors may be subsumed under the headings of feature contrast and feature relevance, respectively. Apparently, when an object differs from its local surroundings (feature contrast), we tend to attend to the object. When the features of an object are relevant to our goals (feature relevance), we also tend to attend to the object. This article

Maria Nordfang, Mads Dyrholm, and Claus Bundesen, Center for Visual Cognition, Department of Psychology, University of Copenhagen, Copenhagen, Denmark.

Correspondence concerning this manuscript should be addressed to Maria Nordfang, Center for Visual Cognition, Department of Psychology, University of Copenhagen, Øster Farimagsgade 2A, DK-1353 Copenhagen, Denmark. E-mail: maria.nordfang@psy.ku.dk is a treatise of the two components of visual attentional weight: the bottom-up, local feature contrast component and the top-down, feature relevance component. The analysis we present is based on psychological experiments with relatively simple stimuli (letters and digits with differently colored local backgrounds), but the principles we derive from our experiments should generalize to visual perception of complex natural scenes.

It is widely accepted that both feature contrast and feature relevance influence attentional selection, and many models of visual attention entail both types of components (e.g., Duncan \& Humphreys, 1989; Müller \& Krummenacher, 2006; Theeuwes, 2010; Wolfe, Cave, \& Franzel, 1989). However, these models differ in their suggested relationship between the two components (cf. Zehetleitner, Goschy, \& Müller, 2012). Some models propose that feature contrast and feature relevance influence attention consecutively (Theeuwes, 2010), some models suggest qualitatively different search modes (e.g., Bacon \& Egeth, 1994), some argue the two components influence attention interdependently (Folk, Remington, \& Johnston, 1992; Found \& Müller, 1996; Zehetleitner et al., 2012), and some propose an additive relationship between the two components (Wolfe, 1994; Wolfe et al., 1989). A central part of this debate has revolved around the initial processing of new stimuli, often exemplified by the onset of a new display on a computer screen or by the first few hundred milliseconds of visual processing after a new fixation. One theoretical view states that initial allocation of attention is determined solely by the physical properties of the stimuli, such that stimuli that differ from their surroundings automatically attract attention (Belopolsky, Zwaan, Theeuwes, \& Kramer, 2007; Theeuwes, 1991, 2004, 2010). Another theoretical view states that attention is directed 
according to our current goals, and by this view, the attentional effect of contrast depends on the relevance of contrast to our goals (e.g., Bacon \& Egeth, 1994; Folk et al., 1992; also see Bundesen, 1990). This discussion has been going on for more than 20 years.

In this article, we investigate the roles of feature contrast and feature relevance in initial visual selection by experimental analysis and computational modeling. Our experimental approach differs from the majority of studies in the previous literature on the topic, as we used post-masked, briefly presented stimulus displays with multiple, simultaneously presented targets, and the participants were not required to make speeded responses. We identified the (bottom-up) feature contrast and the (top-down) feature relevance components of attentional weight by analyzing our experimental data by use of Bundesen's (1990) computational theory of visual attention (TVA). As we shall see, the results suggested a far-reaching generalization of the weight equation of TVA and provided insight into the mechanisms of top-down and bottom-up components of attentional weight.

\section{Previous Studies}

Through the past decades, one of the most influential experimental paradigms for investigating visual selectivity has been the additional singleton paradigm, introduced by Theeuwes (1991). In this paradigm, participants are requested to search for a particular element that is specified as the odd-one-out within a defined feature dimension (e.g., shape) and to respond to the orientation of a line segment located inside this so-called target singleton element. On some trials, an additional singleton is present. The additional singleton is defined by another feature dimension than the target feature dimension (e.g., color). When the contrast of the additional singleton is sufficiently high, reaction times to the target singleton are significantly prolonged (Theeuwes, 1992). These results have been repeatedly replicated (Bacon \& Egeth, 1994; Ester \& Awh, 2008; Theeuwes, 2004), but there is considerable disagreement as to their interpretation.

On one side, it has been argued that data from the additional singleton experiments support the view that local feature contrast determines the initial allocation of attention. As a proponent of this interpretation, Theeuwes (e.g., 1991, 2010) has argued that selection of visual information is done in two stages. At the first stage, selection is driven only by feature contrast such that the element with the highest contrast is the first to be selected, the element with the second highest contrast is the second to be selected, and so on (cf. Theeuwes, 1992; Theeuwes \& Burger, 1998). According to this view, relevance-directed allocation of attention is not possible before the second stage, which supposedly corresponds to approximately $150-175 \mathrm{~ms}$ after the stimuli have been presented (e.g., Theeuwes, 2010, p. 88). Thus, the time taken to find a target element increases in the presence of elements with a higher contrast. A substantial amount of data collected using the additional singleton paradigm has confirmed that search times to find a target singleton increase when a distractor singleton with a sufficiently high local contrast is present (e.g., Theeuwes, 1991, 1992, 2004, 2010).

On the other side, several researchers have opposed the view that initial attentional selection is determined entirely by local feature contrast. These researchers disagreed that the data from the additional singleton experiments show that initial attention is con- trast bound (Bacon \& Egeth, 1994; Folk \& Remington, 1998; Folk et al., 1992; Folk, Remington, \& Wright, 1994; Leber \& Egeth, 2006). They argued that because the target in the additional singleton experiments is a singleton itself, it is contingent with the task to pay attention to singletons. Hence, the additional nontarget singleton is not irrelevant to the task (Bacon \& Egeth, 1994; Folk et al., 1992, 1994). It has been proposed that participants in the additional singleton experiments are in a singleton detection mode in which all singletons, irrespective of the feature dimension in which they are unique, are prioritized (Bacon \& Egeth, 1994; Leber \& Egeth, 2006). Such a strategy is likely to be fairly effortless, and it allows the participant to locate the target. However, when objects with unique features are prioritized, the additional singleton will attract more attention than other nontargets and thereby prolong the time taken to locate the target singleton.

In experiments in which the additional singleton task is modified such that participants can no longer perform the task by means of singleton detection mode (e.g., when the target is a singleton on only a few of the trials), the effect of the additional singleton seems to vanish (Bacon \& Egeth, 1994; Folk \& Remington, 1998; Folk et al., 1992; Leber \& Egeth, 2006). Furthermore, several studies have varied contrast and relevance independently (e.g., Folk \& Annett, 1994; Jonides \& Yantis, 1988; Yantis \& Egeth, 1999) and under these conditions found no effects of static feature singletons. These results agree with the view that static local feature contrast influences initial allocation of attention if, and only if, the contrast signal is relevant to the current task. More recently, the notion of qualitatively different search modes, as proposed by Bacon and Egeth (1994), has been questioned by studies showing gradual top-down modulation of the effect of the additional singleton (Müller, Geyer, Zehetleitner, \& Krummenacher, 2009; Zehetleitner et al., 2012), which have argued that bottom-up and top-down components influence visual attention continuously and interdependently.

All of the above studies agree that the effect of the additional singleton is modulated by top-down components. However, another interpretation of Bacon, Egeth, Folk, Müller, and colleagues' data has been proposed. It has been suggested that the lacking effect of the additional singleton in the above-mentioned studies is due to the small size and the fast disengagement of a spatial attentional window (e.g., Belopolsky \& Theeuwes, 2010; Belopolsky et al., 2007; Theeuwes, 2010). According to this hypothesis, the size of the attentional window depends on the difficulty of the search task. When search becomes difficult, the size of the attentional window is reduced. Thus, when the search task is hard (e.g., Bacon \& Egeth, 1994, Experiments 2 and 3; Jonides \& Yantis, 1988, Experiments 1 and 2), the distracting additional singleton falls outside the narrow window and therefore has no effect on attention (Belopolsky \& Theeuwes, 2010; Belopolsky et al., 2007; Theeuwes, 2004). Furthermore, when the additional singleton is sufficiently different from the target, the attentional window can be disengaged from the distracting singleton and reallocated to the target location very quickly (Belopolsky et al., 2007; Theeuwes, 2010). The disengagement may happen so fast that the attentional allocation to the distracting singleton is indiscernible in reaction times when there is a $150-\mathrm{ms}$ interval between the onset of the distracting singleton and the target display, as in the study by Folk et al. (1992; see Theeuwes, 2010). The validity of these hypothesized mechanisms has been challenged (Folk \& Remington, 2010; 
Müller et al., 2009; Müller, Töllner, Zehetleitner, Rangelov, \& Krummenacher, 2010), and the question of whether initial attentional allocation is determined by relevance, contrast, or both, remains unresolved.

In general, it is striking that the experiments finding support of contrast-dependent attentional selection have tended to disregard the relevance of the contrast, whereas the studies with results consistent with a complete dependency on relevance largely have neglected the strength of the feature contrast. Furthermore, most of the above-mentioned studies have used relatively long, responseterminated stimulus presentations and reaction time as the main dependent variable (for reaction time experiments with fixed exposure duration, see Folk et al., 1992; Theeuwes \& Burger, 1998). As emphasized by Santee and Egeth (1982) and Ester and Awh (2008), performance in such resource-limited conditions reflects not only perceptual but also postperceptual processing, including response selection. By contrast, in a data-limited task in which participants are instructed to make an unspeeded response to target items presented for only a brief period of time, performance may reflect the perceptual processing with higher fidelity (Ester \& Awh, 2008; Santee \& Egeth, 1982). The brief exposures may ensure that performance is limited by the initial encoding of the stimulus display, and the use of unspeeded responses may reduce the likelihood of errors in response selection (or other processes). In view of these considerations, a new approach by use of datalimited, unspeeded-response tasks seems warranted in order to determine the roles of contrast and relevance in initial attentional selection.

\section{A New, Irrelevant-Singleton Paradigm}

To disentangle effects of local feature contrast and relevance to the observer's goals, we need experiments that vary contrast and relevance systematically and independently from each other, while leaving little doubt on whether processing pertains to one or other purported stage. For this purpose, we implemented a new experimental method, introducing color singletons into a partial report task in which color was irrelevant, stimulus displays were briefly presented and post-masked, multiple targets were present, and the dependent variable was accuracy of report. The task was to report the letters from an array of letters (targets) and digits (distractors). On some trials a singleton color was present. The singleton color was the only singleton feature in the displays and as such presented a strong local feature contrast. However, the visibility of the element with the singleton color was the same as the visibility of the remaining, nonsingleton elements. Furthermore, the singleton color was entirely irrelevant to the task. The probability that a color singleton was a target was exactly the same as the probability that a nonsingleton was a target. In addition, targets could not be located on the basis of any type of local feature contrast, so the participants could not perform the task by searching for singletons (cf. Bacon \& Egeth, 1994). These manipulations led to an experimental setup that allowed for disentanglement of effects of contrast from effects of relevance in processing of briefly exposed displays. Importantly, the setup also made it possible to evaluate whether the effect of the local feature contrast of an element (color singleton vs. nonsingleton) depended on the relevance of the element (target vs. distractor).

\section{TVA-Based Predictions}

We analyzed the results of our partial report experiments by use of Bundesen's (1990) theory of visual attention (TVA). At the core of TVA are two equations: the rate equation and the weight equation. The rate equation describes the rate (categorizations/s) at which a specific categorization of an object in the visual field (object $x$ belongs to category $i$ or, equivalently, object $x$ has feature $i$ ) is encoded into visual short term memory (VSTM). This rate is given by Equation 1 of TVA,

$$
v(x, i)=\eta(x, i) \beta_{i} \frac{w_{x}}{\sum_{z \in S} w_{z}},
$$

where $\eta(x, i)$ is the strength of the sensory evidence that object $x$ belongs to category $i, \beta_{i}$ is the perceptual bias associated with category $i$ (i.e., the inclination to categorize objects as members of category $i$ ), and $w_{x} / \sum_{z \in S} w_{z}$ is the relative attentional weight of object $x$ (i.e., the ratio between the attentional weight allocated to object $x$ and the sum of attentional weights across all objects in the visual field). The weight equation describes the amount of attentional weight allocated to an object, and is given by Equation 2 of TVA,

$$
w_{x}=\sum_{j \in R} \eta(x, j) \pi_{j},
$$

where $\eta(x, j)$ is the strength of the sensory evidence that object $x$ belongs to category $j$, and $\pi_{j}$ is the pertinence of category $j$ (i.e., the inclination to categorize objects that are members of category $j$; for further details, see Bundesen, 1990; Bundesen, Habekost, \& Kyllingsbæk, 2005, 2011). Taken together, the two equations describe how $v(x, i)$ is a function of $\eta, \beta$, and $\pi$ values.

TVA has proven to be a powerful tool in analyzing visual attention and the mechanisms that underlie attentional selection, and the theory has been supported by a large body of empirical evidence (Bundesen, 1990, 1998; Bundesen \& Habekost, 2008; Bundesen et al., 2005, 2011; see also Logan, 1996, 2002; Logan \& Gordon, 2001). By fitting behavioral data with a mathematical model based on TVA, it is possible to estimate many parameters of attention such as the attentional weight $(w)$ of each of the different types of items, minimum effective exposure duration $\left(t_{0} \mathrm{~ms}\right)$, processing capacity $(C$ categorizations/s), and storage capacity of VSTM ( $K$ items; Duncan et al., 1999; see Habekost \& Starrfelt, 2009, for a review of TVA-based patient studies). In the current study, we fitted the data by a naive TVA-based model: a model that did not impose any prior assumptions on how the attentional weights of the different element types related to each other. During the estimation process, we let attentional weights of all types of elements in the experiments vary freely. Thus, it was possible to investigate differences between the attentional weights of targets and distractors and of singletons and nonsingletons without making prior assumptions on the way the attentional weights of these elements would be related to each other. Hence, via fitting the empirical data by a mathematical model based on TVA, it was possible to analyze not only if there were effects of relevance and contrast on initial allocation of attention but also how relevance and contrast played together. 


\section{Additive Model of Singleton Weights}

According to traditional TVA, changes in local feature contrast influence attentional selection via changes in $\eta$ values. Consider a simple example. Suppose a red object $x$ becomes surrounded by green objects in space or time. The color contrast between $x$ and the surrounding green objects increases both the strength of the sensory evidence that $x$ is red, $\eta(x$, red $)$ and the strength of the sensory evidence that $x$ is an object with local contrast to its surroundings, $\eta(x$, contrast $)$. The increase in $\eta(x$, red $)$ has two immediate consequences: (a) an increase in $v(x$, red) if the bias toward categorizing objects as red $\left(\beta_{\text {red }}\right)$ is positive and (b) an increase in the attentional weight of $x\left(w_{x}\right)$ if the pertinence of red $\left(\pi_{\text {red }}\right)$ is positive. Similarly, the increase in $\eta(x$, contrast $)$ has two immediate consequences: (a) an increase in $v$ ( $x$, contrast) if the bias toward noticing contrast $\left(\beta_{\text {contrast }}\right)$ is positive and (b) an increase in the attentional weight of $x\left(w_{x}\right)$ if the pertinence of contrast $\left(\pi_{\text {contrast }}\right)$ is positive. A positive pertinence for contrast corresponds to Bacon and Egeth's (1994) proposed singleton detection mode for visual search tasks.

Note that the increase in $v(x$, red $)$ and $v(x$, contrast $)$ increases the probability that $x$ is seen as a red object and as an object with local contrast to its surroundings, respectively. Neither of these effects would be expected to be important in a partial report experiment in which color and contrast are task irrelevant. However, for any feature $i$ with a positive bias $\left(\beta_{i}\right)$, an increase in the attentional weight of $x\left(w_{x}\right)$ increases the probability that $x$ is seen as an object with feature $i$ (e.g., as a letter of a certain type, such as an A). Given that color is irrelevant, $\pi_{\text {red }}$ would be expected to be 0 , such that changes in attentional weight due to changes in $\eta(x$, red $)$ can be ignored. But if $\pi_{\text {contrast }}>0$ in our partial report experiment, an increase in $\eta(x$, contrast $)$ by a certain amount, $\Delta \eta(x$, contrast $)$, should increase the attentional weight of $x\left(w_{x}\right)$ by $\Delta \eta(x$, contrast $)$ $\pi_{\text {contrast }}$ (see Equation 2). This increment should be the same regardless of the prior size of $w_{x}$ and regardless of the feature relevance of $x$ (e.g., whether $x$ is a target or a distractor). Thus, we obtain an additive model of singleton weights. The model predicts that, other things equal, changing the status of an object $x$ from nonsingleton to singleton increases the attentional weight of the object, if $\pi_{\text {contrast }}>0$, by adding an increment, which is a constant independent of the prior size of $w_{x}$.

\section{Experiment 1A}

Our first aim was to test whether both contrast and relevance or just one of the two factors determines the attentional weight allocated to a stimulus in a briefly exposed display. Second, if we found a joint influence of contrast and relevance on the attentional weights, we wished to specify how the two factors contributed to initial attentional selection.

\section{Method}

Participants. Twelve young adults, six women and six men, participated as paid volunteers. Two participants were left out of the analysis: one woman and one man. One of them was left out due to excessive guessing, ${ }^{1}$ and the other was omitted due to misunderstanding of the task. ${ }^{2}$ After exclusion of the two participants, mean age was 26 years, with a range of 23 to 29. All participants had normal or corrected to normal eyesight, and no participants reported any history of color blindness or other visual deficiencies.

Apparatus. The stimuli were presented on a 20-in. CRT screen at a refresh rate of $100 \mathrm{~Hz}$. The resolution was $1,024 \times 768$ pixels. The experiment was run on a ZITECH personal computer using E-prime 1.1 (Psychology Software Tools) on Windows XP. Participants were freely viewing the screen at a distance of approximately $80 \mathrm{~cm}$ in a dimly lit room. Responses were made using a standard PC keyboard.

Stimuli. Participants reported as many letters as possible from an array of six letters (number of targets, $T$ ) and zero, two, or four digits (number of distractors, $D$ ). The six letters were randomly drawn, without replacement, from the set of all the consonants excluding $Y$, which is a vowel in the Danish alphabet. The digits were randomly drawn from the set of numerals from 1 to 9 without replacement. All characters were written in Arial font.

The stimulus displays had a dark gray background color (cf. Table 1 for RGB values and Weber contrasts). All stimulus characters were white and framed by a white circular outline (see Figure 1 for an example of a display). The background color within the white character circle was the same as the display background color (i.e., dark gray) for all stimuli except the color singletons. If an element was a color singleton, the fill color of its character circle differed from the background color of the display and the fill color of the other stimulus elements. The singleton color was randomly chosen from a set of six colors that had the same luminance as the background color of the display and the nonsingleton elements (see Table 1), which made the luminance difference between the white character and the circle fill colors the same for singleton as for nonsingleton elements. Thus, the readability of the stimulus characters was assumed to be the same for all background colors. This assumption was verified in Experiment $1 \mathrm{~B}$.

The distribution of experimental trials with different types of stimuli (different numbers of targets and distractors and different occurrences of singletons) was kept constant in successive blocks of 180 trials (see Table 2). On each trial, at most one color singleton was present. The probability that a singleton element contained a letter was $5 / 7$, which was exactly the same as the probability that a randomly chosen nonsingleton element contained a letter. Furthermore, across all trials, both the probability that a randomly chosen target was a singleton and the probability that a randomly chosen distractor was a singleton were $1 / 12$. Trials without a singleton provided a baseline.

The stimulus elements were presented on the periphery of an imaginary circle, with a radius of approximately 2 degrees of visual angle, centered on a fixation cross. The character circles subtended approximately 0.8 degrees of visual angle, and each character approximately $0.4 \times 0.3$ degree. The stimulus elements were presented on the imaginary circle in any of 12 positions,

\footnotetext{
${ }^{1}$ On $44.3 \%$ of the trials, the participant reported one or more letters that had not been presented. Across all participants, the mean percentage of trials with erroneous reports was $11.0 \%$. The excluded participant deviated by more than two standard deviations from this. All other participants were well within one standard deviation.

${ }^{2}$ The participant reported both letters and digits instead of only the letters, as specified in both the written and oral instructions.
} 
Table 1

$R G B$ Values for the Six Singleton Colors and the Gray Background Color and Weber Contrasts for the White Stimulus Characters, in Experiments $1 A$ and $1 B$

\begin{tabular}{lrrrc}
\hline \multicolumn{1}{c}{ Color } & $\mathrm{R}$ & $\mathrm{G}$ & \multicolumn{1}{c}{$\mathrm{B}$} & Weber contrast \\
\hline Red & 103 & 0 & 0 & 40.33 \\
Green & 0 & 71 & 0 & 40.33 \\
Blue & 0 & 0 & 135 & 40.33 \\
Turquoise & 0 & 60 & 90 & 40.33 \\
Lilac & 85 & 0 & 90 & 40.33 \\
Brown & 85 & 55 & 0 & 40.33 \\
Gray & 60 & 60 & 60 & 40.33 \\
\hline
\end{tabular}

Note. $\mathrm{RGB}=$ red/green/blue color coordinates.

resembling an analog clock. No positions were occupied by more than one element at any one time, and the stimulus positions were drawn at random for each trial.

Each stimulus display was followed by a masking display (see Figure 1). In the masking display, a gray/white random dot noise pattern equal in size to character circles was shown in each of the 12 positions, regardless of whether the position had been occupied by an element in the preceding stimulus display. The masks were identical for all stimuli and were drawn manually in Paint Shop Pro 7.
Procedure. Participants were initially presented with a fixation cross, which remained on the screen for the whole stimulus presentation until participants were to respond. Participants were instructed to fixate before starting each trial and to stay fixated throughout the trial. When the participants felt properly fixated and ready to start the trial, they initiated the trial by pressing enter. With a latency of $100 \mathrm{~ms}$, the stimulus display was shown for 10 , $20,40,70,110$, or $200 \mathrm{~ms}$. The stimulus display was immediately followed by a masking display, exposed for $500 \mathrm{~ms}$ (see Figure 1 for an example of the trial sequence).

Participants were instructed to accurately report as many letters from the stimulus display as possible and to ignore the digits. The order of the reported letters was irrelevant, and the participants could take as long time as desired when reporting the letters. Reports were made by entering the letters on the keyboard. The reported characters were presented on the screen on keypress. When finished with their report, participants pressed space, and the fixation cross reappeared. This indicated the start of a new trial sequence, which the participants again initiated, when ready, by pressing enter.

Each participant performed in four experimental sessions of 900 trials each (corresponding to approximately 45 minutes per session). Each session consisted of five immediately successive blocks of 180 trials. Each trial type was defined by its specific combination of singleton presence, display size, and exposure

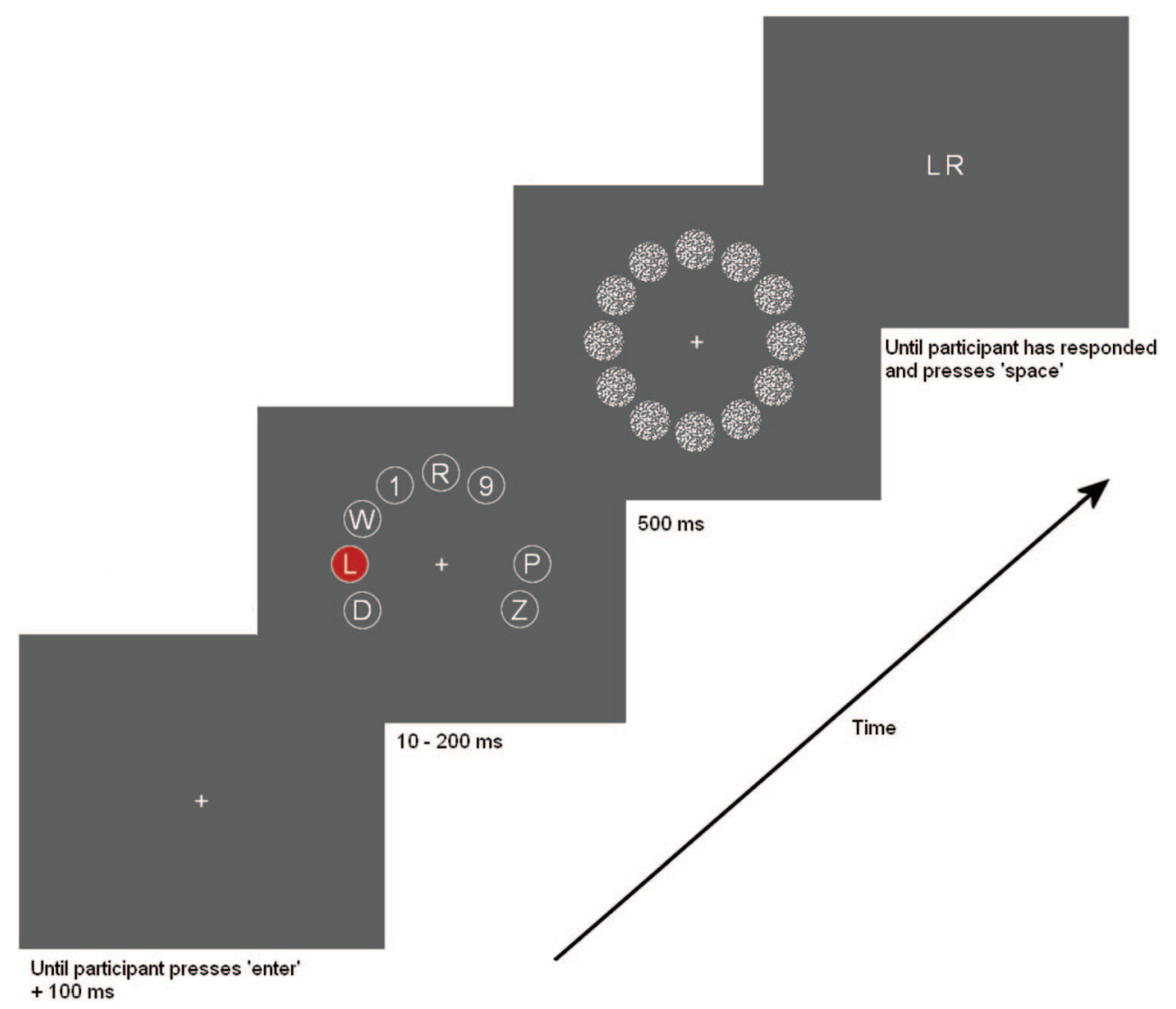

Figure 1. Flowchart of a trial in Experiment 1A. The stimulus display shows a singleton target (a letter on a red background), five other targets (letters), and two distractors (digits). 
Table 2

Number Trials of Different Types at Each Exposure Duration in Each Block of 180 Trials in Experiment 1A

\begin{tabular}{|c|c|c|c|c|c|c|c|c|}
\hline Display size & $\begin{array}{l}\text { Singleton } \\
\text { condition }\end{array}$ & $\begin{array}{c}\# \\
\text { trials }\end{array}$ & \# Ts & $\begin{array}{c}\text { \# T } \\
\text { singletons }\end{array}$ & \# Ds & $\begin{array}{c}\text { \# D } \\
\text { singletons }\end{array}$ & $\mathrm{P}($ singleton $\mid \mathrm{T})$ & $\mathrm{P}($ singleton $\mathrm{ID})$ \\
\hline \multirow{2}{*}{6 Ts and $0 \mathrm{Ds}$} & No & 3 & 18 & 0 & 0 & 0 & $3 /(18+18)=1 / 12$ & Undefined \\
\hline & $\mathrm{T}$ & 3 & 18 & 3 & 0 & 0 & & \\
\hline \multirow[t]{3}{*}{6 Ts and 2 Ds } & No & 4 & 24 & 0 & 8 & 0 & $6 /(24+36+12)=1 / 12$ & $2 /(8+12+4)=1 / 12$ \\
\hline & $\mathrm{T}$ & 6 & 36 & 6 & 12 & 0 & & \\
\hline & $\mathrm{D}$ & 2 & 12 & 0 & 4 & 2 & & \\
\hline \multirow[t]{3}{*}{6 Ts and 4 Ds } & No & 2 & 12 & 0 & 8 & 0 & $6 /(12+36+24)=1 / 12$ & $4 /(8+24+16)=1 / 12$ \\
\hline & $\mathrm{T}$ & 6 & 36 & 6 & 24 & 0 & & \\
\hline & $\mathrm{D}$ & 4 & 24 & 0 & 16 & 4 & & \\
\hline Total & & & 180 & 15 & 72 & 6 & & \\
\hline $\mathrm{P}($ Tlsingleton $)$ & & $15 /(15$ & -6) $=$ & & & & & \\
\hline $\mathrm{P}(\mathrm{T} \mid$ nonsingleton $)$ & & $(180$ & $15) /[(18$ & $-15)+(7$ & $-6)]=$ & & & \\
\hline
\end{tabular}

Note. $\#=$ number $; \mathrm{T}=\operatorname{target} ; \mathrm{D}=$ distractor; No $=$ no singletons; $\mathrm{P}=$ probability.

duration, and the order of the trial types was randomized within each block.

There was as a minimum a 90 -min break between sessions, and no more than two sessions were performed by the same participant on the same day. Each session began with a short training session, consisting of 45 trials, in which participants were presented with displays without singletons and without distractors at exposure durations of 20, 40, 70, 110, and $200 \mathrm{~ms}$. After each training trial, participants were informed of the number of correctly reported letters and the number of errors they had made on that trial. No feedback was given on the experimental trials.

\section{Results}

Mean number of correctly reported targets. Each participant's mean number of correctly reported targets was analyzed as a function of exposure duration $(10,20,40,70,110$, or $200 \mathrm{~ms})$ for each display size (zero, two, or four distractors) and each singleton condition (no singleton, target singleton, or distractor singleton; see Figure 2). The mean numbers of correctly reported targets were examined by two repeated-measures analyses of variance (ANOVAs). Here and elsewhere, we used a .05 level of significance.

The displays with zero distractors were analyzed by a $6 \times 2$ ANOVA with factors of exposure duration and singleton condition. The ANOVA revealed a main effect of exposure duration, $F(5,45)=184.69, p<.001$. Singleton condition had no significant effect on the mean scores; neither was the interaction Singleton Condition $\times$ Exposure Duration significant.

The displays with two and four distractors were analyzed by a $6 \times 3 \times 2$ ANOVA with factors exposure duration, singleton condition, and display size (two or four distractors). The ANOVA revealed a main effect of exposure duration, $F(5,45)=160.92$,
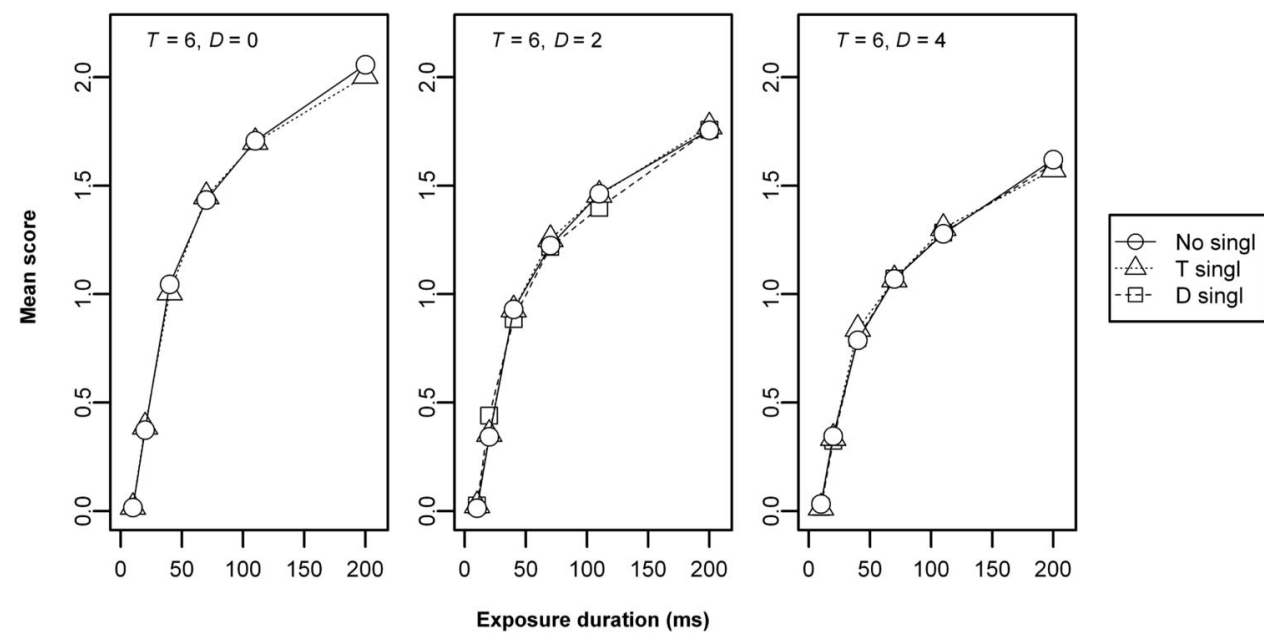

Figure 2. Group mean number of correctly reported targets, for each display size and singleton condition, as a function of exposure duration in Experiment $1 \mathrm{~A}$. $\mathrm{T}=\operatorname{target;} T=$ number of targets; $\mathrm{D}=$ distractor; $D=$ number of distractors; singl $=$ singleton. 
$p<.001$, and display size, $F(1,9)=102.58, p<.001$. Again, singleton condition had no significant effect on the mean scores, and none of the interactions were significant.

The probability of correctly reporting different target types. The probabilities of correctly reporting the different types of targets were analyzed as functions of exposure duration, display size, and target type (nonsingleton targets with no singleton present in the display, nonsingleton targets with a target singleton present in the display, nonsingleton targets with a distractor singleton present in the display, and target singletons; see Figure 3). As with the mean scores, the probabilities of correctly reporting the different target types were examined by two separate repeatedmeasures ANOVAs: one $6 \times 3$ ANOVA with factors exposure duration and target type for displays with zero distractors and one $6 \times 4 \times 2$ repeated-measures ANOVA with factors exposure duration, target type, and display size for displays with two and four distractors. The significant effects of the two ANOVAs are reported in Table 3. The ANOVAs were followed by simple contrast analyses of the comparisons of primary interest, that is, analyses of differences between the probability of correct report for a baseline target (i.e., a target in a display without singletons) and the probability of correct report for other target types.

Overall, the ANOVAs revealed that target type had a significant effect on the probability of whether a given target was correctly reported (cf. Table 3). Thus, the presence of a singleton color significantly influenced the probability of correctly reporting a target. The details of this influence were investigated through the planned contrasts. The contrasts revealed that the singleton targets had a higher probability of being correctly reported than the baseline targets. Furthermore, when a singleton target was present, the nonsingleton targets had a lower probability of being correctly reported than baseline targets. For displays in which a singleton distractor was present, the targets also had a lower probability of being correctly reported than did baseline targets, but the detrimental effect of a singleton distractor was less than the detrimental effect of a singleton target (cf. Table 4 for statistics on the contrasts).

Fitting response probabilities by a naïve model based on TVA. The response probabilities for each participant were fitted by a mathematical model based on TVA by use of a maximum likelihood method (for details, see Dyrholm, Kyllingsbæk, Espeseth, \& Bundesen, 2011; also see Kyllingsbæk, 2006; Shibuya \& Bundesen, 1988). For each participant, minimum effective exposure duration $\left(t_{0}\right)$, processing capacity $(C)$, and a distribution of VSTM capacity ( $K$ distribution) with five free parameters were estimated. The average VSTM capacity $(K)$ for each participant was estimated as the mean of the $K$ distribution. In addition, for each participant, attentional weights $(w)$ for all element types (singleton target, singleton distractor, nonsingleton target, nonsingleton distractor) were estimated at each of the 12 possible stimulus locations. Thus, we estimated a high number of attentional weights, without making any prior assumptions on the way the weights were related to each other. That is, we estimated separate weights for each of the smallest stimulus entities in the displays (each of the four different element types on each of the 12 stimulus locations). In the following, we refer to this model as the naive model. The naive model had 54 free parameters and, not surprisingly, yielded fits that were close to the observed data. ${ }^{3}$ The squared correlations, $r^{2}$, between the observed and predicted mean scores for the 48 different types of trials ranged between .975 and .994 for individual participants; all the correlations were highly significant with $p<.001$. Figure 4 shows an example of observed and predicted mean scores for an individual participant, and Table 5 shows the estimated distribution of $K$, mean $K, t_{0}$, and $C$, for each participant.

Estimates of attentional weight. The mean estimated attentional weights for each stimulus type across all positions and participants are shown in Figure 5. As can be seen, the mean weight of singleton targets was higher than the weight of nonsingleton targets; the weight of singleton distractors was higher than the weight of nonsingleton distractors; and the weights of singleton and nonsingleton targets were higher than the weights of singleton and nonsingleton distractors. Participants' mean estimated weights for each of the four stimulus types were entered into a $2 \times 2$ repeated-measures ANOVA with factors relevance (target, distractor) and contrast (singleton, nonsingleton). The analysis revealed a main effect both of relevance, $F(1,9)=127.52, p<.001$, and of contrast, $F(1,9)=26.68, p<.001$. The interaction Relevance $\times$ Contrast was also significant, $F(1,9)=5.51, p=.043$.

A graphic representation of all the estimated weights for one participant is shown in Figure 6. The attentional weights varied across positions, as can be seen from the individual example of Participant 7 in Figure 6. The distribution of attention across the 12 positions was investigated by individual repeated-measures ANOVAs for each participant across stimulus types with position as factor. The ANOVAs revealed that the patterns of positionrelated variation of attentional weights was consistent across stimulus types; $F(11,33)$ ranged from 5.408 to 19.980 with all $p$ values $<.001$.

For each participant, all estimated singleton weights were plotted against the weights of otherwise similar nonsingleton elements (see Figure 7). As predicted by the additive model of singleton weights, the plotted points fell approximately on a straight line, but contrary to the predictions by the additive model, the slope of the line seemed to be greater than 1. Fitting the points for individual participants by straight lines with freely varying intercepts and slopes (a two-parameter linear model of singleton weights) yielded fairly good fits. The mean $r^{2}$ for the 10 fits was .86 (see the leftmost columns of Table 6 for individual $r^{2}$ values, fitted parameters, and root mean squared deviation [RMSD] for each participant). We also fitted the data by a straight line with a slope fixed at one (a one-parameter additive model of singleton weights) and a straight line with an intercept fixed at zero (a one-parameter multiplicative model of singleton weights). The simple oneparameter models were tested against the two-parameter model by likelihood ratio tests (see Table 6). For six out of the 10 participants, the two-parameter linear model was significantly better than the simple additive model; across all 10 participants, $\chi^{2}(10)=$ $129.29, p<.001$. By contrast, the two-parameter linear model was significantly better than the simple multiplicative model for just one of the 10 participants. For this participant, $\chi^{2}(1)=6.14, p<$ .02 ; across the remaining nine participants, $\chi^{2}(9)=15.21, p>.05$;

\footnotetext{
${ }^{3}$ A total of 48 weight estimates (12 positions $\times$ four element types) on a ratio scale with arbitrary unit, one $t_{0}$ estimate, one distribution of $K$ with five free parameters, and one $C$ estimate.
} 

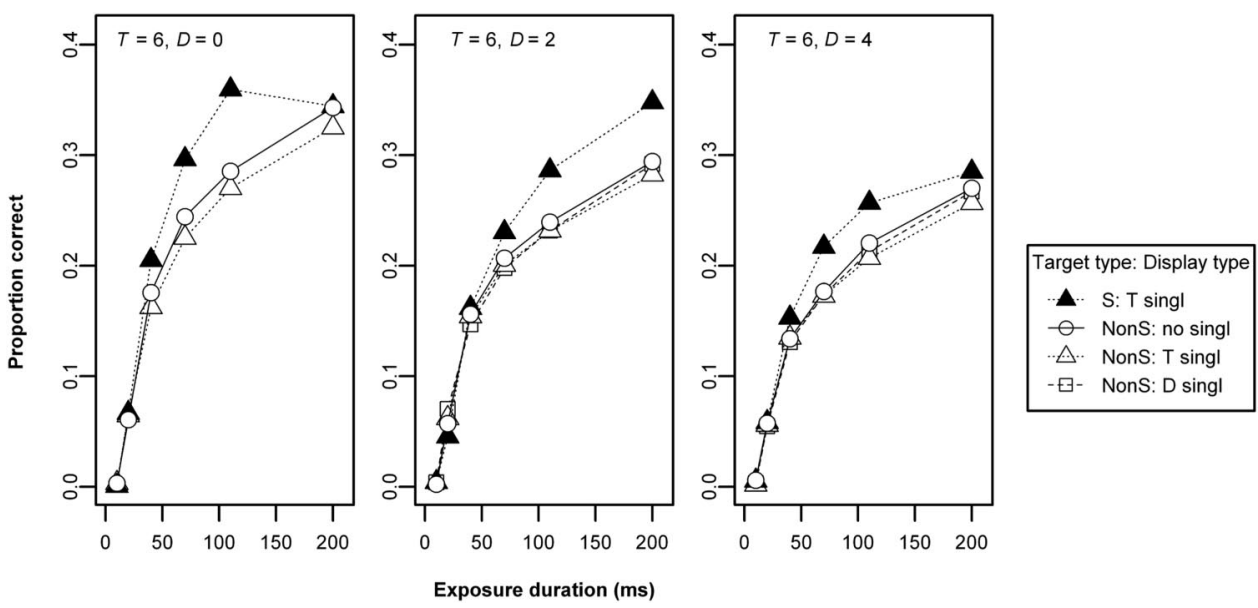

Figure 3. Group mean proportion of correctly reported targets of different types, for each display size, as a function of exposure duration in Experiment 1A. $T=$ number of targets; $D=$ number of distractors; $\mathrm{S}: \mathrm{T}$ singl $=$ the singleton in a display with a target singleton; NonS:no singl = nonsingleton target in a display without singletons; NonS:T singl $=$ nonsingleton target in a display with a target singleton; NonS: $\mathrm{D}$ singl $=$ nonsingleton target in a display with a distractor singleton.

however, in total across all 10 participants, $\chi^{2}(10)=21.36, p=$ .019 .

\section{Discussion}

The results of Experiment 1A showed no significant effect of the presence of a singleton on the mean number of correctly reported targets. Nevertheless, the distribution of processing resources seemed affected by local feature contrast. A singleton target had a significantly higher probability of being correctly reported than a baseline nonsingleton target, and when a nonsingleton target was replaced by a singleton target, the probability of correctly reporting the nonsingleton targets in the same display significantly decreased. Thus, singleton targets appeared to summon more processing resources than nonsingleton targets. The singleton distractors had a similar effect, but it was weaker. When a singleton distractor was present, there was a small but significant tendency for the probability of correctly reporting the nonsingleton targets in the same display to decrease. These results point toward

Table 3

Significant Effects by Analyses of Variance on Probabilities of Correctly Reporting a Given Target in Experiment $1 \mathrm{~A}$

\begin{tabular}{llrrr}
\hline \multicolumn{1}{c}{ Display } & \multicolumn{1}{c}{ Source } & $d f_{\text {num }}$ & $d f_{\text {denom }}$ & \multicolumn{1}{c}{$F$} \\
\hline 6T0D & Exposure duration (ED) & 5 & 45 & $175.92^{* * * *}$ \\
& Target type (TT) & 2 & 18 & $52.74^{* * * *}$ \\
& ED $\times$ TT & 10 & 90 & $7.61^{* * * *}$ \\
6T2D and 6T4D & 5 & 45 & $165.36^{* * * *}$ \\
& ED & 3 & 27 & $11.36^{* * * *}$ \\
& TT & 1 & 9 & $105.49^{* * * *}$ \\
& Display size (DS) & 15 & 135 & $7.27^{* * * *}$ \\
& ED $\times$ TT & 5 & 45 & $18.78^{* * * *}$ \\
\hline
\end{tabular}

Note. $\quad d f=$ degrees of freedom; num $=$ numerator of $F$ ratio; denom $=$ denominator of $F$ ratio; $6 \mathrm{~T} 0 \mathrm{D}=6$ targets and 0 distractors; $6 \mathrm{~T} 2 \mathrm{D}=6$ targets and 2 distractors; 6T4D $=6$ targets and 4 distractors. ***** $p<.001$. a joint influence of contrast and relevance. It appeared that the magnitude of the influence of a strong color contrast depended on the relevance of the element with the contrast.

Effects of contrast and relevance were also reflected in the attentional weights that were estimated by the naive mathematical model based on TVA. The attentional weights for singleton elements were consistently higher than the attentional weights for nonsingletons. The weights also revealed an attentional priority for targets compared with distractors. The estimated attentional weights of the targets were significantly higher than the weights of the distractors, reflecting selectivity consistent with the relevance of the elements. Furthermore, there was a significant interaction between effects of contrast and effects of relevance on the attentional weights. Plotting the estimated singleton weights against the weights of otherwise identical

Table 4

Within-Subject Comparisons of Probabilities of Reporting Different Types of Targets in Various Types of Displays Against the Probability of Reporting a Baseline Target in Experiment 1A

\begin{tabular}{llcrc}
\hline \multicolumn{1}{c}{$\begin{array}{c}\text { Type of } \\
\text { target }\end{array}$} & $\begin{array}{c}\text { Singleton type } \\
\text { of display }\end{array}$ & $\begin{array}{c}\text { Mean difference } \\
\text { from baseline }\end{array}$ & $t(9)$ & $p$ \\
\hline & & $D=0$ \\
Nonsingleton & Target & -0.0093 & -7.67 & $<.001^{* * * *}$ \\
Singleton & Target & 0.0267 & 7.96 & $<.001^{* * *}$ \\
\hline \multicolumn{5}{c}{$D=2$ or 4} \\
Nonsingleton & Target & -0.0047 & -3.38 & $.004^{* * *}$ \\
Nonsingleton & Distractor & -0.0033 & -1.97 & $.040^{*}$ \\
Singleton & Target & 0.0026 & 3.28 & $.005^{* *}$ \\
\hline
\end{tabular}

Note. Displays contained 6 targets and 0,2 , or 4 distractors. A baseline target was a target in a display without any singletons. Directions of the effects were predicted, so testing was one-tailed. $D=$ number of distractors.

${ }^{*} p<.05 . \quad{ }^{* *} p<.01 . \quad{ }^{* * *} p<.001$. 

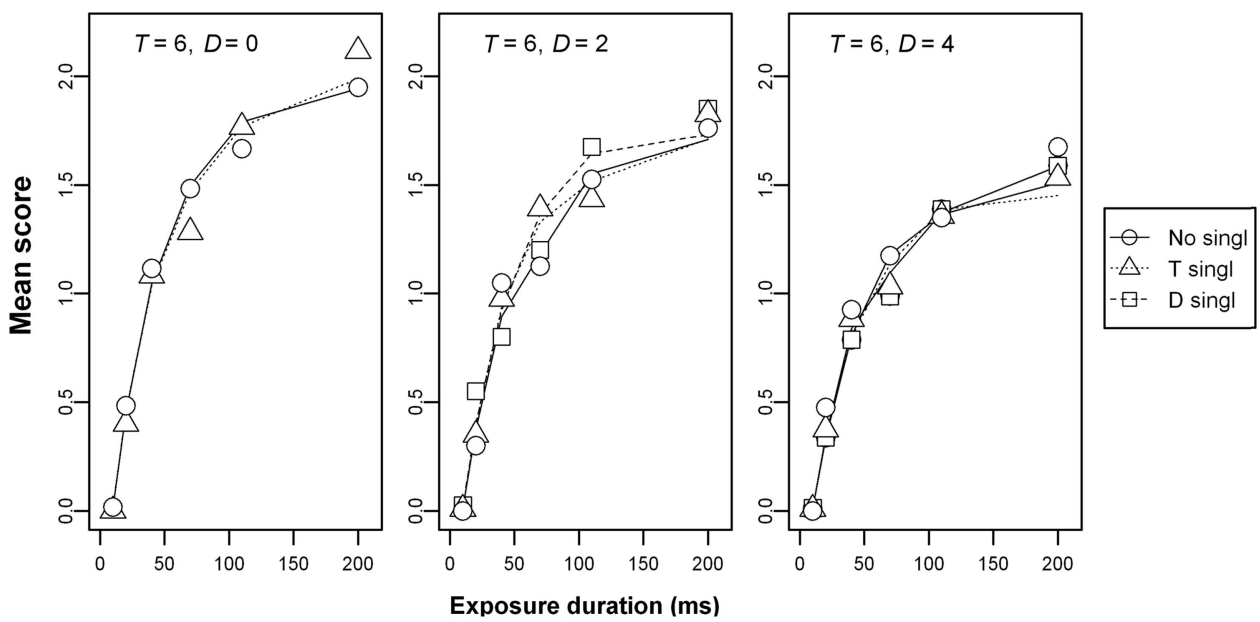

Figure 4. Observed and predicted mean numbers of correctly reported targets for a representative participant (Participant 5), for each display size and singleton condition, as functions of exposure duration in Experiment 1A. $\mathrm{T}=\operatorname{target} ; T=$ number of targets; $\mathrm{D}=$ distractor; $D=$ number of distractors; singl $=$ singleton.

nonsingletons produced points lying on approximately straight lines with slopes greater than one. When we compared three types of linear models-a two-parameter linear model, a oneparameter multiplicative model, and a one-parameter additive model-it was apparent from the likelihood ratio tests that the simple additive model was inferior to the two-parameter linear model. The two-parameter model also fitted significantly better than the simple multiplicative model, but the difference in goodness of fit between these two models was small, and the difference was significant for just one of the 10 participants.

\section{Experiment 1B}

A control experiment was carried out, in order to ensure that the increased probability of correctly reporting the singleton targets compared with the baseline nonsingleton targets was not confounded by differences in readability between singleton and nonsingleton elements. In Experiment 1A, the singleton colors were

Table 5

Parameter Estimates for Each Participant in Experiment $1 \mathrm{~A}$

\begin{tabular}{ccccccccccc}
\hline & \multicolumn{1}{c}{ Probability distribution of $K$} & & & \\
\cline { 2 - 6 } Participant & 1 & 2 & 3 & 4 & 5 & 6 & Mean $K$ & \multicolumn{1}{c}{$t_{0}$} & $C$ \\
\hline 1 & .45 & .49 & .04 & .01 & .01 & .01 & 1.65 & 8.76 & 38.06 \\
2 & .31 & .56 & .12 & .01 & .00 & .00 & 1.85 & 9.50 & 42.72 \\
3 & .11 & .68 & .20 & .00 & .00 & .00 & 2.12 & 18.40 & 45.63 \\
4 & .31 & .53 & .14 & .01 & .01 & .01 & 1.89 & 18.58 & 29.01 \\
5 & .06 & .64 & .28 & .01 & .00 & .00 & 2.27 & 9.80 & 52.72 \\
6 & .09 & .69 & .21 & .01 & .00 & .00 & 2.15 & 9.97 & 48.21 \\
7 & .02 & .30 & .60 & .08 & .00 & .00 & 2.75 & 9.75 & 83.30 \\
8 & .22 & .58 & .18 & .01 & .01 & .01 & 2.01 & 9.32 & 52.99 \\
9 & .18 & .62 & .19 & .00 & .00 & .00 & 2.04 & 7.95 & 70.04 \\
10 & .34 & .44 & .19 & .01 & .01 & .01 & 1.94 & 9.80 & 53.06 \\
\hline
\end{tabular}

Note. The estimates were derived from fits of the data by the naive mathematical model based on the theory of visual attention. $K=$ storage capacity (number of characters); $t_{0}=$ minimum effective exposure duration (in milliseconds); $C=$ processing capacity (characters per second). chosen to be equiluminant with the display background color (and thus also with the background color of the nonsingleton elements). Yet, candela values are not behavioral measures. We wanted to test behaviorally that there was no difference in the readability of the letters when they were presented on a local gray background compared to when they were presented on a local colored background. In Experiment 1B we tested this by presenting the participants with a single target and varying the local background color of the element.

\section{Method}

Participants. The same 10 volunteers as in Experiment $1 \mathrm{~A}$ participated in the experiment.

Apparatus and stimuli. The apparatus and stimulus material were similar to those used in Experiment 1A with the following

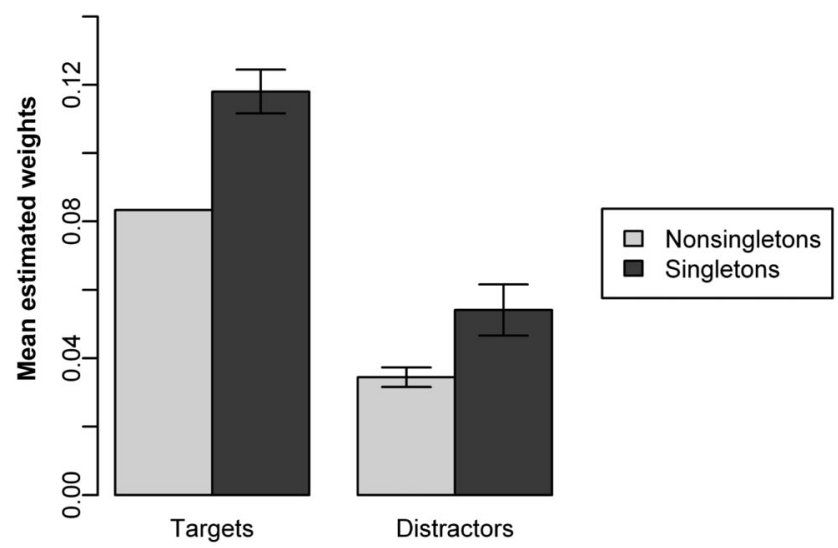

Figure 5. Group means of estimated attentional weights for each stimulus type across all spatial positions in Experiment 1A. Error bars represent standard errors of the means. The weights were normalized so that the sum of nonsingleton target weights equaled 1 for each participant; hence, the error bars for nonsingleton targets equal zero. 

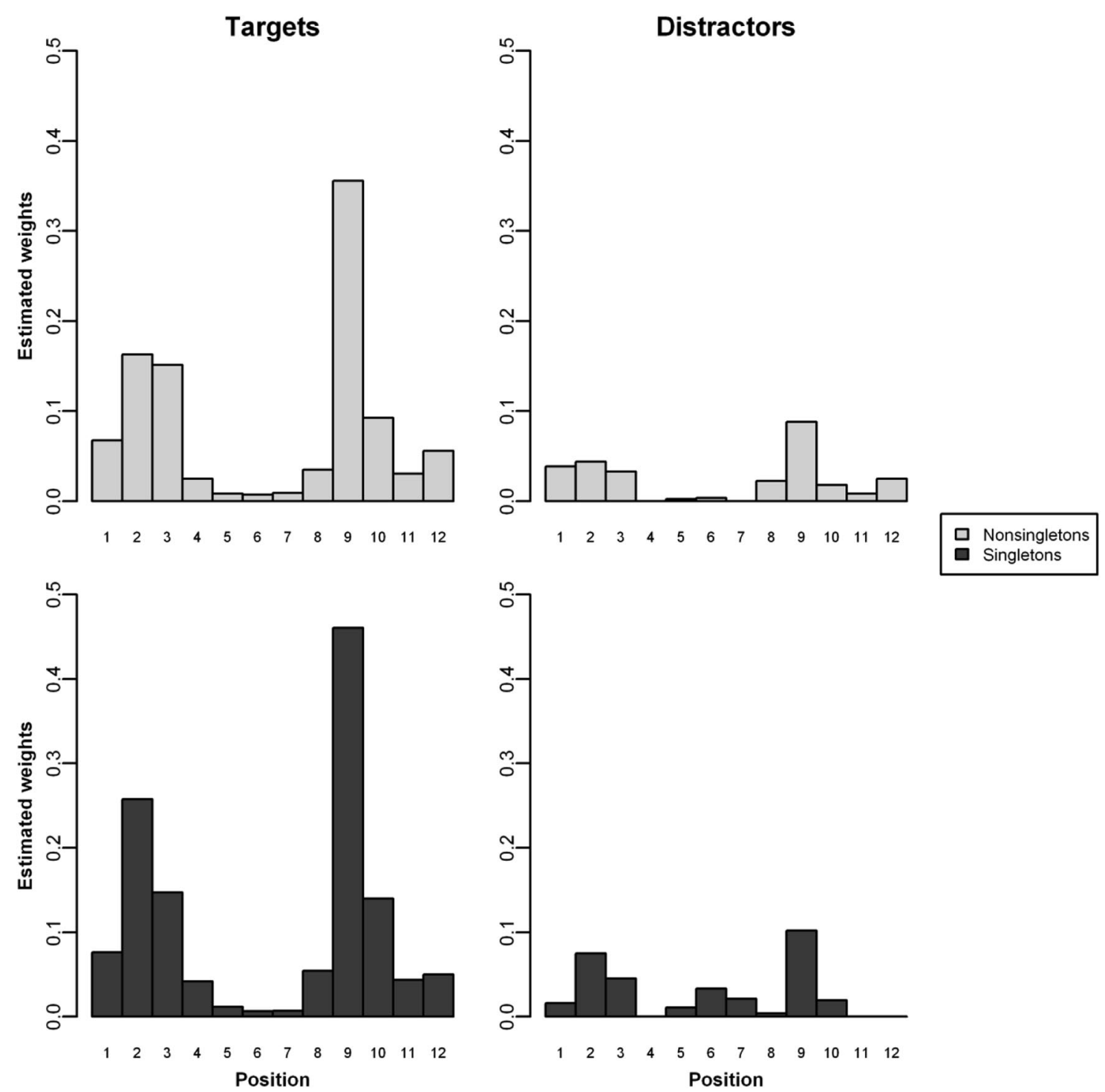

Figure 6. Estimated attentional weights for each stimulus type at each spatial position for a representative participant (Participant 7) in Experiment 1A. The positions are numbered like the hours on the face of an analog clock.

exception: Only a single target and no distractors were presented in each display. The target was randomly chosen on each trial from the same letter set as in Experiment 1A. The background color of the presented stimulus varied. On half the trials, the local background color was the same as the display background color. On the other half of the trials, the local background color varied randomly between the same six colors as were used as singleton colors in Experiment 1A (see Table 1). The target was shown in one of the 12 positions on an imaginary circle similar to the preceding experiment. The position of the target was randomly chosen for each trial.

Procedure. Participants were instructed to report the target as accurately as possible. As in Experiment 1A, participants were initially presented with a fixation cross, which remained on the screen for the whole stimulus presentation until they were to respond. The participants were instructed to fixate the cross before starting each trial and to stay fixated throughout the trial. When properly fixated, participants pressed enter. With a latency of 100 $\mathrm{ms}$ after the keypress, a location cue (a thin line 3/5 the length of the radius of the imaginary circle) pointed toward the location of the upcoming target for $100 \mathrm{~ms}$. Just $300 \mathrm{~ms}$ after the presentation of the location cue, the target appeared at the cued location for 10 , $20,30,50,70,100$, or $130 \mathrm{~ms}$, followed by a 500-ms presentation of a masking display similar to the one used in Experiment 1A. See Figure 8 for an example of a trial sequence.

The participants carried out four sessions of 252 trials (i.e., a total of 1,008 trials) equaling 72 observations per trial type (7 exposure durations $\times 2$ stimulus types). The trials were presented in random order within each session. There was a minimum of a 90-min break between successive sessions, and not more than two 

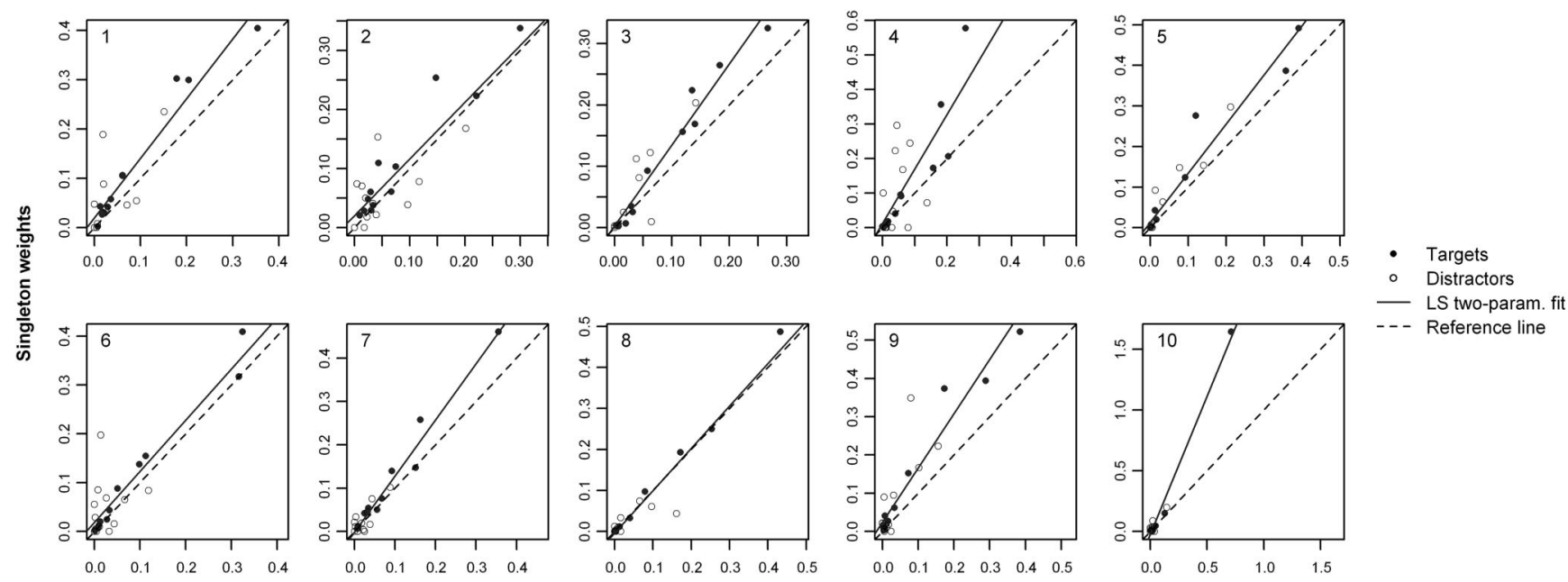

Figure 7. Estimated attentional weights of singleton targets and distractors at different spatial positions plotted against the attentional weights of otherwise similar, nonsingleton targets and distractors at the same positions, for each of the 10 participants in Experiment 1A. For each participant, the data were fitted by a least squares straight line with freely varying slope and intercept (LS two-param. fit). The dashed reference line is $y=x$.

sessions were performed by one participant on the same day. Each session began with a short training phase consisting of 21 trials, to get the participants attuned to the short exposure durations. After each trial in the training phase, participants received onscreen feedback on their report (correct report, erroneous report, or no report).

\section{Results and Discussion}

The mean number of correctly reported targets was analyzed across participants for each stimulus type (gray local background and colored local background) as a function of exposure duration $(10,20,30,50,70,100,130 \mathrm{~ms}$; see Figure 9). The data were examined by a repeated-measures ANOVA with factors exposure duration and stimulus type. The ANOVA revealed a significant main effect of exposure duration, $F(6,54)=124.33, p<.001$. There was no significant effect of stimulus type, $F(1,9)=0.25$, $p=.627$. The interaction Exposure Duration $\times$ Stimulus Type was not significant.

The mean number of correctly reported targets for the two different stimulus types, colored and gray, did not differ significantly from each other in the single target report. The data support the assumption that the effects of contrast found in the main experiment were not confounded by differences in readability between singleton and nonsingleton characters. The characters were equally recognizable on the gray and the colored backgrounds.

Table 6

Best Fits of Each of the Three Linear Models for All Singleton Weights Against Corresponding Nonsingleton Weights for Each Participant in Experiment $1 \mathrm{~A}$

\begin{tabular}{|c|c|c|c|c|c|c|c|c|c|c|c|c|}
\hline \multirow[b]{3}{*}{ Participant } & \multicolumn{4}{|c|}{ Two-parameter model } & \multicolumn{4}{|c|}{ One-parameter multiplicative model } & \multicolumn{4}{|c|}{ One-parameter additive model } \\
\hline & \multirow[b]{2}{*}{ Intercept } & \multirow[b]{2}{*}{ Slope } & \multirow[b]{2}{*}{$r^{2}$} & \multirow[b]{2}{*}{ RMSD } & \multirow[b]{2}{*}{ Slope } & \multirow[b]{2}{*}{ RMSD } & \multicolumn{2}{|c|}{ LRT } & \multirow[b]{2}{*}{ Intercept } & \multirow[b]{2}{*}{ RMSD } & \multicolumn{2}{|c|}{ LRT } \\
\hline & & & & & & & $\chi^{2}(1)$ & $p$ & & & $\chi^{2}(1)$ & $p$ \\
\hline 1 & 0.0198 & 1.209 & .843 & 0.0463 & 1.320 & 0.0465 & 3.13 & .077 & 0.0319 & 0.0470 & 3.56 & .059 \\
\hline 2 & 0.0201 & 0.960 & .761 & 0.0409 & 1.091 & 0.0436 & 3.05 & .081 & 0.0174 & 0.0410 & 0.13 & .717 \\
\hline 3 & 0.0027 & 1.321 & .925 & 0.0262 & 1.340 & 0.0263 & 0.15 & .693 & 0.0210 & 0.0345 & 13.15 & $<.001^{* * * *}$ \\
\hline 4 & 0.0117 & 1.579 & .601 & 0.0896 & 1.663 & 0.0900 & 0.22 & .639 & 0.0492 & 0.0983 & 4.44 & $.035^{*}$ \\
\hline 5 & 0.0131 & 1.211 & .938 & 0.0339 & 1.263 & 0.0357 & 2.55 & .110 & 0.0262 & 0.0410 & 9.10 & $.002^{* *}$ \\
\hline 6 & 0.0182 & 1.047 & .803 & 0.0452 & 1.142 & 0.0477 & 2.64 & .105 & 0.0208 & 0.0453 & 0.195 & .689 \\
\hline 7 & -0.0014 & 1.294 & .957 & 0.0209 & 1.285 & 0.0210 & 0.08 & .784 & 0.0142 & 0.0307 & 18.37 & $<.001^{* * * * *}$ \\
\hline 8 & -0.0043 & 1.034 & .932 & 0.0285 & 1.016 & 0.0287 & 0.41 & .520 & -0.0024 & 0.0287 & 0.35 & .553 \\
\hline 9 & 0.0239 & 1.419 & .858 & 0.0559 & 1.529 & 0.0595 & 2.98 & .084 & 0.0490 & 0.0691 & 10.15 & $.001^{* *}$ \\
\hline 10 & -0.0251 & 2.273 & .982 & 0.0434 & 2.214 & 0.0493 & 6.14 & $.013^{*}$ & 0.0442 & 0.1859 & 69.84 & $<.001^{* * * *}$ \\
\hline
\end{tabular}

Note. Root mean squared deviation (RMSD) is noted for comparisons between the models. The one-parameter models are tested against the two-parameter model by likelihood ratio tests (LRT); for both comparisons, the nested model differs from the standard model by one degree of freedom; $\chi^{2}$ and $p$ statistics are provided for these tests.

${ }^{*} p<.05 .^{* * *} p<.01 .^{* * * *} p<.001$. 


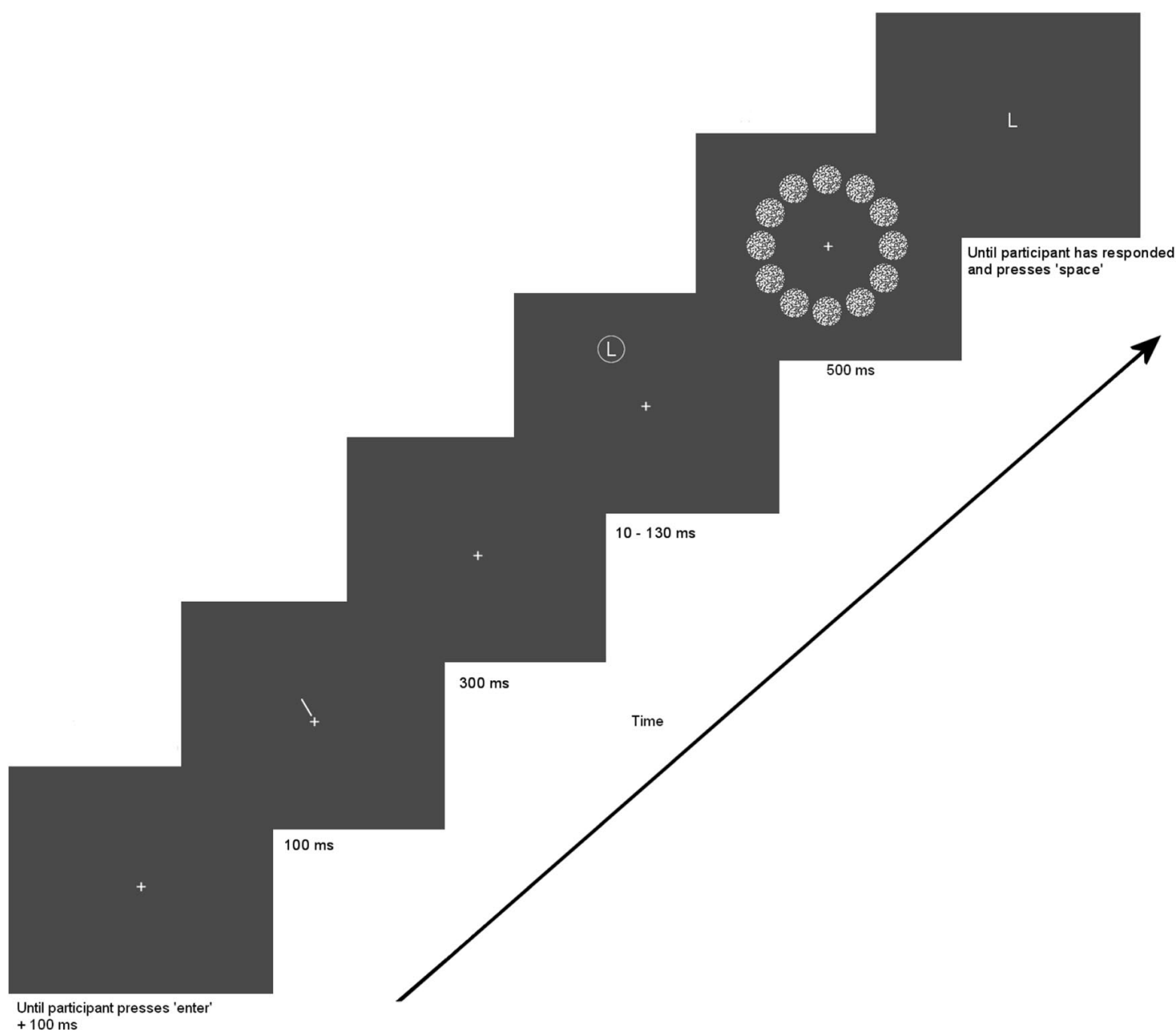

Figure 8. Flowchart of a trial in Experiment 1B. The stimulus display shows a condition with a gray local background color.

\section{Discussion of Experiments 1A and 1B}

The results of Experiments 1A and 1B show that an element with a uniquely colored local background attracts more attention than a corresponding element that is not uniquely colored. This difference cannot be ascribed to differences in readability of the target letter dependent on the local background color. Nor has the unique color any relation to the task at hand. Taken together, the results are consistent with the notion that a color singleton element receives higher attentional weight both when the element is a target and when it is a distractor. At the same time, the data suggest that attention is allocated consistent with feature relevance (higher attentional weights on targets than distractors). Thus, initial visual selectivity appears to be determined by both components of attentional weight: contrast and relevance.

We investigated how the strong contrast signal affected the attentional weights in Experiment 1A. The data suggested a linear relation between the attentional weight of a singleton and the weight of an otherwise similar nonsingleton. Such a linear relation can be modeled by a one-parameter additive model, a one-parameter multiplicative model, or a full two-parameter linear model. The simple additive model is the only one that is entirely consistent with the traditional weight equation of TVA (Equation 2). A good fit by the additive model would suggest that the pertinence of local feature contrast was positive. However, the setup of Experiment 1A discouraged a positive pertinence of contrast, and likelihood ratio tests revealed that the simple additive model was inferior to the two-parameter linear model. The two-parameter model also fitted significantly better than the simple multiplicative model, but the difference in goodness of fit between these two models was small, and the difference was significant for just one of the 10 participants.

New weight equation. In the two-parameter linear model of singleton weights, the weight of a singleton element is related to the weight of an otherwise similar nonsingleton element by both a multiplicative and an additive constant. In order to account for such a relationship we propose the following generalization of the weight equation of TVA:

$$
w_{x}=\kappa_{x} \sum_{j \in R} \eta(x, j) \pi_{j},
$$

where $\kappa_{x}$ is the strength of the local feature contrast of object $x$, and the summation across $j$ subsumes $\eta(x$, contrast $) \pi_{\text {contrast }}$, which is 


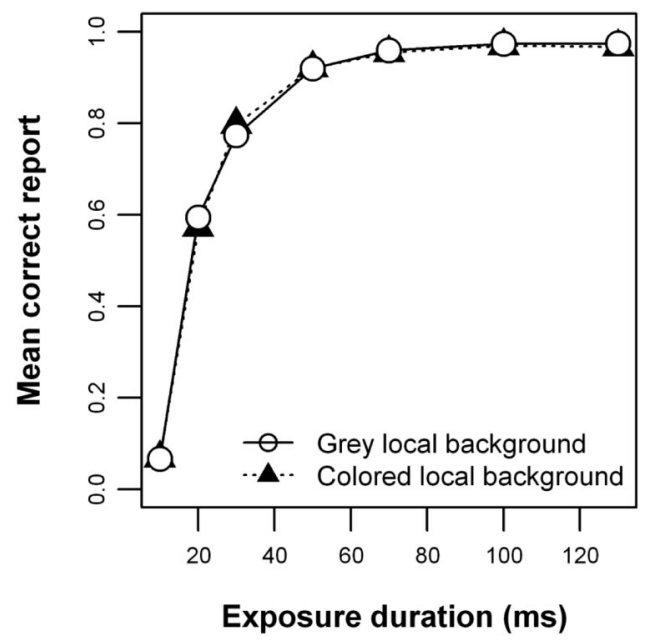

Figure 9. Group mean number of correctly reported targets for each stimulus type as a function of exposure duration in Experiment 1B.

the product of the strength of the sensory evidence that object $x$ belongs to the category high local feature contrast and the pertinence of the category high local feature contrast. Without loss of generality, we can assume that $\kappa_{\text {nonsingleton }}$ equals one. Hence, by Equation 3, attentional weights of singleton elements are given as functions of the attentional weights of otherwise similar nonsingleton elements by the linear equation

$$
w_{\text {singleton }}=m\left(w_{\text {nonsingleton }}+a\right),
$$

where

$$
m=\kappa_{\text {singleton }}
$$

and

$$
\begin{aligned}
a=\eta(\text { singleton, contrast }) & \pi_{\text {contrast }} \\
& -\eta(\text { nonsingleton, contrast }) \pi_{\text {contrast }},
\end{aligned}
$$

$\eta$ (singleton, contrast) being the strength of the sensory evidence that a singleton element has a high local feature contrast, and $\eta$ (nonsingleton, contrast) being the strength of the sensory evidence that a nonsingleton element has a high local feature contrast.

The one-parameter multiplicative model of singleton weights is a special case of the two-parameter linear model. In the oneparameter multiplicative model, the weight of a singleton element is related to the weight of an otherwise similar nonsingleton element by a single multiplicative constant. To account for such a simple multiplicative relationship, we propose Equation 3 with the constraint that, under the conditions of our experiment, the pertinence value of the category of high local feature contrast $\left(\pi_{\text {contrast }}\right)$ equals zero. Given that $\pi_{\text {contrast }}=0$, Equation 6 implies that Equation 4 holds with $a=0$ such that

$$
w_{\text {singleton }}=m w_{\text {nonsingleton }},
$$

where $m=\kappa_{\text {singleton }}$.

We devised two new mathematical models for the probability distributions of correct responses based on TVA, models TVA ma and $\mathrm{TVA}_{m}$, by use of Equation 3 with $\pi_{\text {contrast }} \geq 0$ and $\pi_{\text {contrast }}=$
0 , respectively. Apart from the computation of the attentional weights, both models were similar to the naive model. For Experiment 1A, TVA ${ }_{m a}$ had 32 free parameters (one five-parameter distribution of $K$, parameters $t_{0}$ and $C, 12$ nonsingleton target weights whose sum was fixed at a value of 1,12 nonsingleton distractor weights, the multiplicative constant $m$, and the additive constant $a$ ). $\mathrm{TVA}_{m}$ had 31 free parameters (same parameters as TVA $_{m a}$ but no additive constant). The two new models fitted the data very well; correlations between each of the participants' observed mean scores for the 48 experimental conditions and the predicted mean scores for each of the two models ranged from $r^{2}=.935$ to $r^{2}=.994$, with all $p$ values $<.001$. By the likelihood ratio tests reported in Table $7, \mathrm{TVA}_{m a}$ did not fit the data significantly better than $\mathrm{TVA}_{m}, \chi^{2}(10)=17.10, p=.072$. On the other hand, let TVA $\mathrm{TV}_{m \mid m}={ }_{1}$ be $\mathrm{TVA}_{m}$ with the added constraint that $m=$ 1. Reassuringly, $\mathrm{TVA}_{m}$ fit the data significantly better than TVA $_{m \mid m=1}$. The difference was highly significant, $\chi^{2}(10)=$ $214.49, p \ll .001$ (see Table 7 for individual statistics).

In summary, the response probabilities observed in Experiment 1A were well fitted by mathematical models based on TVA using our generalized weight equation (Equation 3) instead of the original weight equation (Equation 2). Fits based on the assumption that $\pi_{\text {contrast }}=0\left(\right.$ model $\left.\mathrm{TVA}_{m}\right)$ were pitted against fits based on the more general assumption that $\pi_{\text {contrast }} \geq 0\left(\right.$ model TVA $\mathrm{Ta}_{\text {a }}$ ). Fits based on the assumption that $\pi_{\text {contrast }} \geq 0$ were not significantly better than fits based on the assumption that $\pi_{\text {contrast }}=0$ (see Table 7), suggesting that in Experiment $1 \mathrm{~A}, \pi_{\text {contrast }}$ was close to 0 . However, across participants in Experiment 1A, the relationship between the singleton versus nonsingleton weights estimated by the naive model was significantly better fitted by the twoparameter linear model than by the one-parameter multiplicative model (see Table 6), suggesting that $\pi_{\text {contrast }}>0$. Presumably, $\pi_{\text {contrast }}$ was positive for one or more participants in Experiment $1 \mathrm{~A}$ but was not much greater than 0 .

\section{Experiment 2A}

In Experiment 2A the singleton colors were manipulated so that there were two distinct types of color singletons: weak color

\begin{tabular}{|c|c|c|c|c|}
\hline \multirow[b]{2}{*}{ Participant } & \multicolumn{2}{|c|}{ TVA $_{m a}$ vs. TVA $m$} & \multicolumn{2}{|c|}{ TVA $_{m}$ vs. TVA TVIm=1 } \\
\hline & $\chi^{2}(1)$ & $p$ & $\chi^{2}(1)$ & $p$ \\
\hline 1 & 0.00 & 1.00 & 82.11 & $<.001^{* * *}$ \\
\hline 2 & 3.97 & .05 & 0.70 & .40 \\
\hline 3 & 2.73 & .10 & 30.57 & $<.001^{* * * *}$ \\
\hline 4 & 0.00 & .99 & 16.60 & $<.001^{\text {**** }}$ \\
\hline 5 & 0.34 & .56 & 11.06 & $<.001^{\text {**** }}$ \\
\hline 6 & 0.17 & .68 & 19.74 & $<.001^{\text {***** }}$ \\
\hline 7 & 0.18 & .67 & 26.37 & $<.001^{\text {**** }}$ \\
\hline 8 & 3.48 & .06 & 10.88 & $<.001^{\text {**** }}$ \\
\hline 9 & 4.26 & $.04^{*}$ & 16.42 & $<.001^{\text {***** }}$ \\
\hline 10 & 1.97 & .16 & 0.03 & .86 \\
\hline
\end{tabular}
singletons (with a unique color that was only slightly different

Table 7

Likelihood Ratio Comparisons of Fits by TVA ${ }_{m a}$ Versus $T V A_{m}$ and $T V A_{m}$ Versus $T V A_{m \mid m=1}$, for Experiment $1 A$

Note. $\quad \mathrm{TVA}=$ theory of visual attention.

${ }^{*} p<.05$. ${ }^{* * * *} p<.001$. 
from the background color of the display) and strong color singletons (with a unique color that deviated strongly from the background color of the display). If the effects of the singletons we found in Experiment 1A pertained to the strength of the local feature contrasts, the weak color singletons would be expected to have a smaller effect on initial attentional allocation than would the strong singletons. Such an effect of the strength of local feature contrast on attention has recently been demonstrated by salience modulation of the latency and amplitude of the posteriorcontralateral negativity (PCN; Töllner, Zehetleitner, Gramann, \& Müller, 2011). In setup, Experiment 2A was similar to Experiment $1 \mathrm{~A}$, but minor changes were made to accommodate for the new color manipulation and to improve the experimental design by presenting the stimuli equally spaced around the perimeter of the circular display.

\section{Method}

Participants. Six young adults, four women and two men, served as paid volunteers. Participants' mean age was 23 years, with a range of 21 to 26 . All participants had normal or corrected to normal eyesight, and no participants reported any history of color blindness or other visual deficiencies.

Apparatus. The apparatus was the same as that used in Experiment $1 \mathrm{~A}$.

Stimuli. The stimulus setup in Experiment 2A was comparable to that used in Experiment 1A with the following exceptions. The stimulus array always consisted of eight elements combined in one of three ways: two targets and six distractors, four targets and four distractors, or six targets and two distractors. Thus, the targetdistractor configuration was varied without varying the display size. Summed across all displays, the total number of target elements equaled the total number of distractor elements. Thus, contrary to Experiment $1 \mathrm{~A}$, no redundant trials were needed in order to keep the probability that a singleton contained a target equal to the probability that a singleton contained a distractor (cf. Table 2). The probability that a nonsingleton element contained a target was .5 , which was exactly the same as the probability that a singleton element contained a target. In total across all the displays, $1 / 10$ of the elements were singletons. On some trials no singleton was present. These trials were used as a baseline.

The stimuli were presented at eight evenly spaced positions on the periphery of an imaginary circle with a radius of approximately 2.3 degrees of visual angle. All eight positions were occupied by a stimulus element in all displays.

The stimuli used in Experiment 2A were similar but not identical to the stimuli used in Experiments $1 \mathrm{~A}$ and $1 \mathrm{~B}$. The stimulus displays had a light gray background color. All stimulus characters were black and were framed by a black circular outline. The background color inside the black character circle was the same as the display background color (i.e., light gray) for all stimuli except the color singleton elements.

Only two colors were used as singleton colors. The singleton color could be either a strong, saturated red or a weak, unsaturated red. All element fill colors were calibrated such that the perceptual contrast between each of the three colors and the black character color was the same based on a behavioral measure. See Experiment $2 \mathrm{~B}$ for the exact calibration procedure and Table 8 for RGB values and Weber contrasts for all the used colors. The stimulus characters subtended approximately $0.6 \times 0.4$ degree of visual angle and the character circles subtended 1.2 degrees.

Each stimulus display was followed by a masking display consisting of gray/black/red random dot noise patterns presented on each of the eight stimulus positions in the display. The masks were identical for all stimuli, and they were equal in size to the black outlined character circles. The masks were drawn in Paint Shop Pro 7.

Procedure. The individual trials followed the same trial sequence as in Experiment 1A, with the following exception: After the participants had responded, a feedback screen with the number of correctly reported letters and the number of erroneously reported letters written in dark gray was presented for $500 \mathrm{~ms}$.

Each participant served in 8,100 experimental trials equaling 90 observations per data point per participant (6 exposure durations $X$ 3 target-distractor configurations $\times 5$ singleton conditions). The trials were presented in random order. The 8,100 trials were divided into nine sessions of 900 trials each. Each session began with a short training session of 30 trials, presenting all targetdistractor configurations and all singleton conditions at the two longest exposure durations. After every 100 trials, participants were encouraged to rest their eyes and take a little break. Participants took part in at most two sessions per day, and two same-day sessions were always separated by a break of at least 15 minutes.

\section{Results and Discussion}

Mean number of correctly reported targets. The mean numbers of correctly reported targets were analyzed across participants as a function of target-distractor configuration (6 targets and 2 distractors; 4 targets and 4 distractors; 2 targets and 6 distractors), exposure duration (10, 20, 40, 70, 110, $200 \mathrm{~ms})$, and singleton condition (strong target singleton, weak target singleton, strong distractor singleton, weak distractor singleton, or no singleton; see Figure 10) by a repeated-measures ANOVA. The ANOVA revealed main effects of target-distractor configuration, $F(2$, $10)=132.85, p<.001$; exposure duration, $F(5,25)=267.83$, $p<.001$; and singleton condition, $F(4,20)=3.96, p=.016$. All the two-way interactions were significant; however, the three-way interaction was not significant.

To investigate further the significant effect of the singletons on the mean number of correctly reported targets, we compared the four singleton conditions to the baseline condition in which there was no singleton in the display. There was a significant difference between the strong target singleton condition and the baseline, $F(1$, $5)=12.245, p=.017, M_{\text {strong target singleton }}=1.184, M_{\text {baseline }}=$

Table 8

$R G B$ Values for the Two Singleton Colors and the Gray Background Color and Weber Contrasts for the Black Stimulus Characters, in Experiments $2 A$ and $2 B$

\begin{tabular}{lrrrr}
\hline \multicolumn{1}{c}{ Color } & $\mathrm{R}$ & $\mathrm{G}$ & \multicolumn{1}{c}{$\mathrm{B}$} & Weber contrast \\
\hline Strong red & 235 & 0 & 0 & -0.981 \\
Weak red & 153 & 128 & 108 & -0.976 \\
Gray & 144 & 132 & 113 & -0.977 \\
\hline
\end{tabular}

Note. $\mathrm{RGB}=$ red/green/blue color coordinates. 

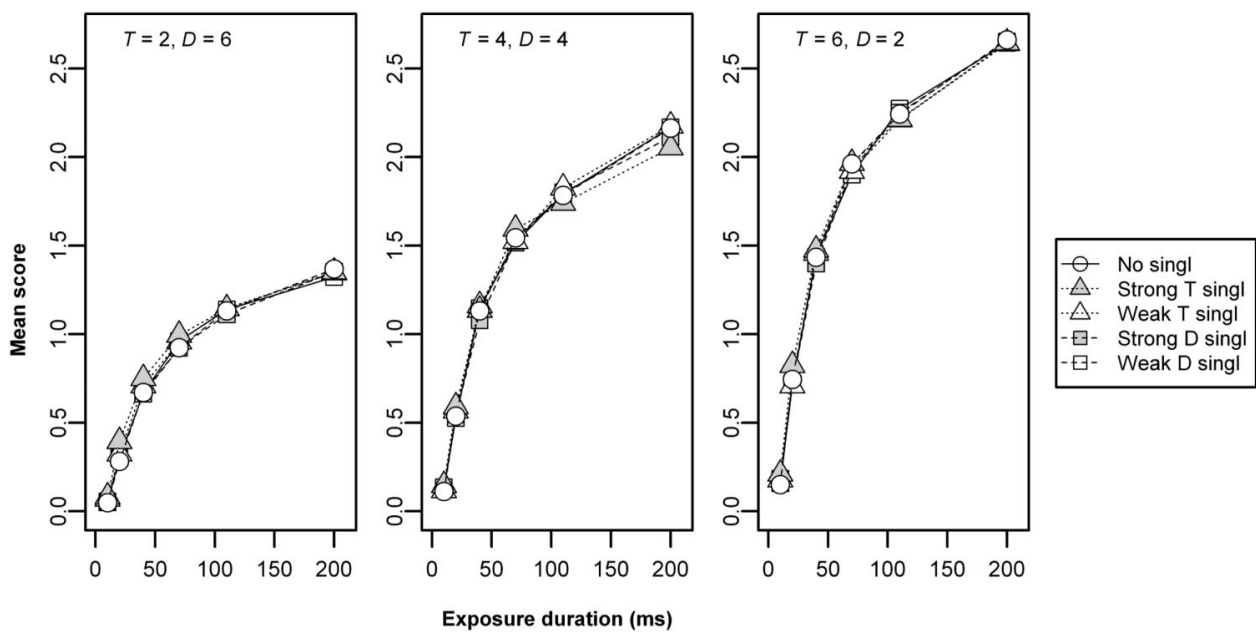

Figure 10. Group mean number of correctly reported targets, for each target-distractor configuration and singleton condition, as a function of exposure duration in Experiment $2 \mathrm{~A} . \mathrm{T}=\operatorname{target} ; T=$ number of targets; $\mathrm{D}=$ distractor; $D=$ number of distractors; $\operatorname{singl}=$ singleton.

1.160. None of the other singleton conditions was significantly different from the baseline.

The probability of correctly reporting different target types. The probability of correctly reporting a target was analyzed for each of the seven target types (nonsingleton targets with no singleton present in the display, nonsingleton targets with a strong target singleton present in the display, nonsingleton targets with a weak target singleton present in the display, targets with a strong distractor singleton present in the display, targets with a weak distractor singleton present in the display, strong target singletons, and weak target singletons) as a function of target-distractor configuration and exposure duration. Figure 11 shows the mean proportion of times a target was correctly reported across participants for the different target types. Individual participants' probability to correctly report a target was examined by a repeated-measures 6 (exposure durations) $\times 7$ (target types) $\times 3$ (target-distractor configurations) ANOVA. All significant effects from this ANOVA are reported in the upper rows of Table 9. As in Experiment 1A, the ANOVA revealed a significant main effect of target type. Furthermore, the proportion of stimulus elements that were targets, in these displays with constant set size, had a significant influence on the probability to correctly report a given target.

We repeated the ANOVA excluding exposure durations above $110 \mathrm{~ms}$. The resulting 5 (exposure durations) $\times 7$ (target types) $\times$ 3 (target-distractor configurations) ANOVA revealed a significant main effect of both target type and target-distractor configuration (see the middle rows of Table 9 for report of all significant effects).
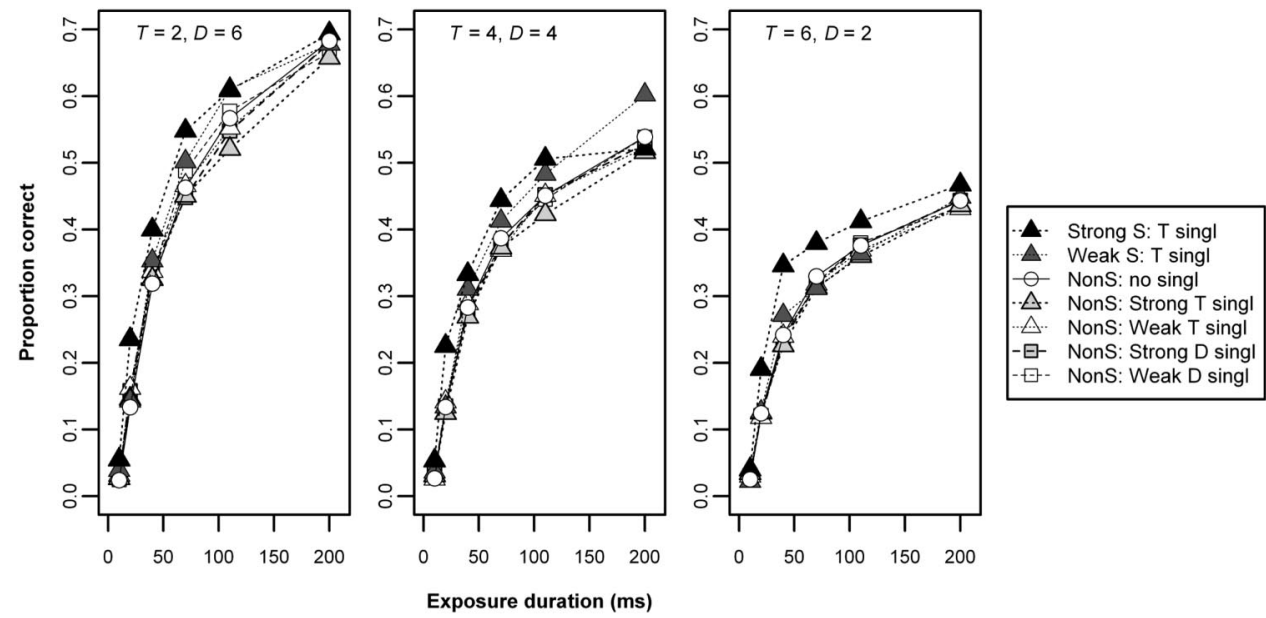

Figure 11. Group mean proportion of correctly reported targets of different types, for each target-distractor configuration, as a function of exposure duration in Experiment 2A. $T=$ number of targets; $D=$ number of distractors; S:T singl = the singleton in a display with a target singleton; NonS:no singl = nonsingleton target in a display without singletons; NonS:T singl = nonsingleton target in a display with a target singleton; NonS: $\mathrm{D} \operatorname{singl}=$ nonsingleton target in a display with a distractor singleton. 
The proportion of correctly reported baseline targets (targets in displays without singletons) was compared with the proportion of correct report for all the other target types across all six exposure durations. The contrasts revealed that the probability to report a strong singleton target was higher than the baseline, $t(5)=3.03$, $p=.015, M_{\text {strong target singleton }}=0.359, M_{\text {baseline }}=0.308$, as was the probability to report a weak singleton target, $t(5)=2.62, p=$ $.024, M_{\text {weak target singleton }}=0.325$. None of the other target types was reported with a probability significantly different from the baseline.

The difference between strong and weak singletons was further investigated. The probability of correctly reporting singleton targets was analyzed separately, across all exposure durations, by a 6 (exposure durations) $\times 2$ (target types) $\times 3$ (target-distractor configurations) ANOVA, excluding the five nonsingleton target types. There was a significant main effect of target type (strong vs. weak singleton). Also, the main effect of target-distractor configuration was again highly significant (see the lower rows of Table 9 for report of all significant effects).

Fitting response probabilities by a naive model based on TVA. As in Experiment 1A, the response probabilities observed for each individual participant were fitted by a naive mathematical model based on Bundesen's (1990) TVA. For each participant, the distribution of VSTM capacity (distribution of $K$ ), minimum effective exposure duration $\left(t_{0}\right)$, and speed of encoding $(C)$ were estimated. Also, attentional weights $(w)$ for all element types (strong singleton target, weak singleton target, nonsingleton target, strong singleton distractor, weak singleton distractor, nonsingleton distractor) were estimated at each of the eight stimulus positions, resulting in a model with a total of 54 free parameters. The model yielded close fits to the observed data. For the 90 different experimental conditions, the correlations between the observed mean scores and the predicted mean scores from the fits for each participant ranged from $r^{2}=.990$ to $r^{2}=.996$. All the correlations were highly significant, with $p<.001$. See Figure 12 for an example of observed and predicted mean scores for Participant 5 and Table 10 for $K$ distribution, mean $K, t_{0}$ and $C$ estimates for each participant.
Estimates of attentional weight. The mean estimated attentional weights for each stimulus type averaged across all positions and all participants are shown in Figure 13. As can be seen, the mean weight of strong singleton targets was higher than the weight of weak singleton targets, which in turn was higher than the weight of nonsingleton targets. Also, the mean weight of strong singleton distractors was higher than the weight of weak singleton distractors, which in turn was higher than the weight of nonsingleton distractors. Furthermore, the weights of strong, weak, and nonsingleton targets were higher than the weights of strong, weak, and nonsingleton distractors. The mean estimated weights for each stimulus type for each participant were entered into a $2 \times 3$ repeated-measures ANOVA with the factors relevance (target, distractor) and contrast (strong singleton, weak singleton, nonsingleton). The analysis revealed a significant main effect both of relevance, $F(1,5)=115.91, p<.001$, and of contrast, $F(2,10)=$ $5.95, p=.020$. Also, the interaction Relevance $\times$ Contrast was significant, $F(2,10)=4.42, p=.042$.

A graphic representation of all the estimated weights for one participant (Participant 1) is shown in Figure 14. Repeatedmeasures ANOVAs were carried out for each participant, across stimulus types and with position as the factor. The ANOVAs revealed that the pattern of position-related variation of attentional weights was consistent across stimulus types; $F(7,35)$ ranged from 6.327 to 20.521 , with all $p$ values $<.001$.

For each of the six participants, all estimated strong-singleton weights (weights of targets and distractors at each of the eight stimulus positions) were plotted against the estimates of the weights of the corresponding nonsingletons. The same procedure was followed for all estimated weak-singleton weights (see Figure 15). Fitting the points by straight lines yielded reasonably good fits (the $r^{2}$ for the 12 fits ranged from .826 to .974; see Table 11 for individual $r^{2}$ values, fitted parameters, and RMSDs). We tested whether the fits of the two-parameter linear model were driven by either a simple multiplicative or a simple additive model. Hence, we also fitted all the data by straight lines with intercepts fixed at zero and by straight lines with slopes fixed at one. The oneparameter fits were tested against the two-parameter fits by like-

Table 9

Significant Effects by Analyses of Variance on Probabilities of Correctly Reporting a Given Target in Experiment $2 \mathrm{~A}$

\begin{tabular}{|c|c|c|c|c|}
\hline ANOVA & Source & $d f_{\text {num }}$ & $d f_{\text {denom }}$ & $F$ \\
\hline \multirow{5}{*}{$\begin{array}{l}6 \text { (exposure durations) } \times 7 \text { (target types) } \times 3 \text { (target-distractor } \\
\text { configurations) }\end{array}$} & Exposure duration (ED) & 5 & 25 & $4.94^{* * * *}$ \\
\hline & Target type (TT) & 6 & 30 & $7.21^{* * * *}$ \\
\hline & TD configuration & 2 & 10 & $77.79^{* * * *}$ \\
\hline & $\mathrm{ED} \times \mathrm{TT}$ & 30 & 150 & $2.03^{* *}$ \\
\hline & $\mathrm{ED} \times \mathrm{TD}$ & 12 & 60 & $1.13^{* * * * *}$ \\
\hline \multirow{5}{*}{$\begin{array}{l}5 \text { (exposure durations) } \times 7 \text { (target types) } \times 3 \text { (target-distractor } \\
\text { configurations) }\end{array}$} & ED & 4 & 20 & $241.55^{* * *}$ \\
\hline & TT & 6 & 30 & $12.70^{* * * *}$ \\
\hline & $\mathrm{TD}$ & 2 & 10 & $70^{* * * *}$ \\
\hline & $\mathrm{ED} \times \mathrm{TT}$ & 24 & 120 & $1.88^{*}$ \\
\hline & $\mathrm{ED} \times \mathrm{TD}$ & 8 & 40 & $0.069^{* * * *}$ \\
\hline \multirow{6}{*}{$\begin{array}{l}6 \text { (exposure durations) } \times 2 \text { (target types) } \times 3 \text { (target-distractor } \\
\text { configurations) }\end{array}$} & ED & 5 & 25 & $129.61^{* * * *}$ \\
\hline & TT & 1 & 5 & $7.46^{*}$ \\
\hline & TD & 2 & 10 & $29.93^{* * * *}$ \\
\hline & $\mathrm{ED} \times \mathrm{TT}$ & 5 & 25 & $3.74^{*}$ \\
\hline & $\mathrm{ED} \times \mathrm{TD}$ & 10 & 50 & $19.76^{* * * *}$ \\
\hline & $\mathrm{TT} \times \mathrm{TD}$ & 2 & 10 & $5.98^{*}$ \\
\hline
\end{tabular}

Note. $\quad$ ANOVA $=$ analysis of variance; $d f=$ degrees of freedom; num $=$ numerator; denom $=$ denominator; $\mathrm{TD}=$ target - distractor.

${ }^{*} p<.05 .{ }^{* * *} p<.01 .^{* * * *} p<.001$. 

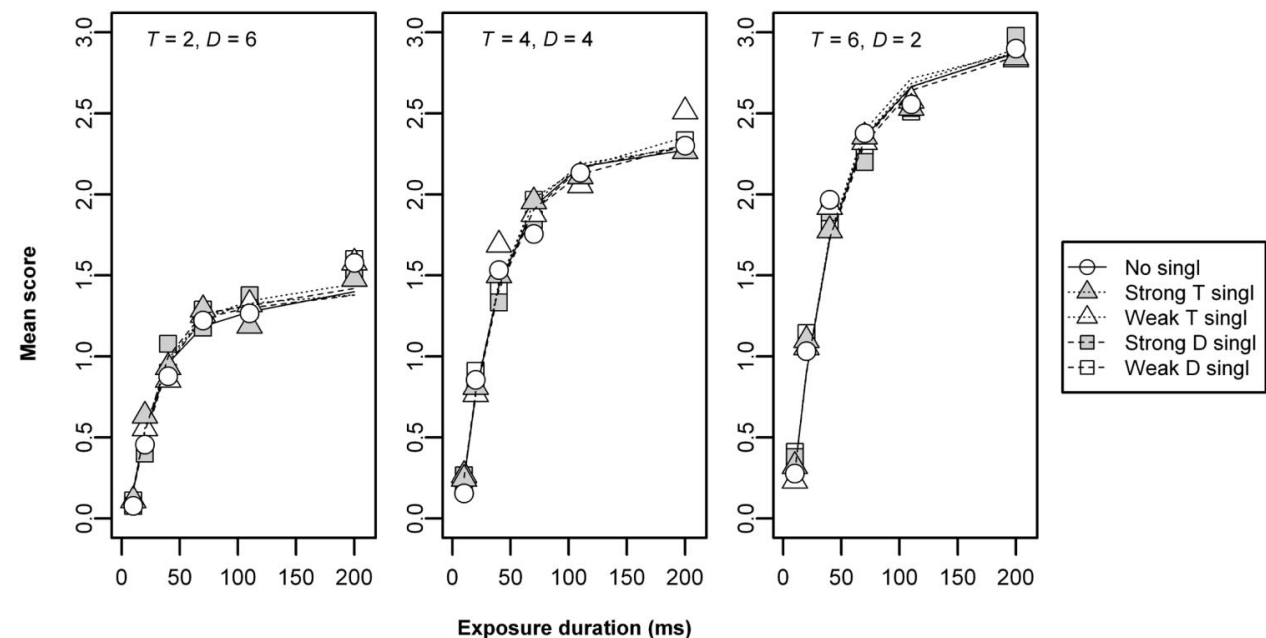

Figure 12. Observed and predicted mean numbers of correctly reported targets for a representative participant (Participant 5), for each target-distractor configuration and singleton condition, as functions of exposure duration in Experiment 2A. $\mathrm{T}=$ target; $T=$ number of targets; $\mathrm{D}=$ distractor; $D=$ number of distractors; singl $=$ singleton.

lihood ratio tests. None of the 12 two-parameter fits were significantly better than the corresponding multiplicative fit, whereas five of the 12 two-parameter fits were significantly better than the corresponding additive fits (see Table 11). The overall chi-square tests showed that the two-parameter linear model fitted significantly better than the additive model, $\chi^{2}(12)=26.73, p<.001$, but not significantly better than the multiplicative model, $\chi^{2}(12)=$ $12.78, p=.386$.

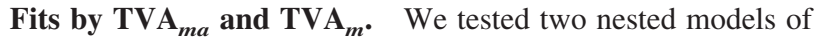
the response probabilities based on TVA: TVA $m a$ and $\mathrm{TVA}_{m}$, which applied Equation 3 with $\pi_{\text {contrast }} \geq 0$ and $\pi_{\text {contrast }}=0$, respectively. For either model, two values of parameter $m$ (i.e., $\left.\kappa_{\text {singleton }}\right)$ were estimated-one value for the strong singletons and one for the weak singletons. For TVA ${ }_{m a}$, in which $\pi_{\text {contrast }} \geq 0$, parameter $a$ was also estimated separately for weak and strong singletons. Apart from the computation of the attentional weights, the parameters of both models were similar to the naive model of Experiment 2A. For Experiment 2A, TVA ${ }_{m a}$ had 26 free parameters (one five-parameter distribution of $K$, parameters $t_{0}$ and $C$, eight nonsingleton target weights whose sum was fixed at a value

Table 10

Parameter Estimates for Each Participant in Experiment $2 \mathrm{~A}$

\begin{tabular}{cccccccccrr}
\hline & \multicolumn{1}{c}{ Probability distribution of $K$} & & & \\
\cline { 2 - 6 } Participant & 1 & 2 & 3 & 4 & 5 & 6 & Mean $K$ & $t_{O}$ & \multicolumn{1}{c}{$C$} \\
\hline 1 & .06 & .66 & .28 & .00 & .00 & .00 & 2.24 & 9.00 & 48.89 \\
2 & .00 & .25 & .41 & .29 & .02 & .02 & 3.15 & 1.39 & 48.78 \\
3 & .02 & .23 & .52 & .19 & .03 & .01 & 2.00 & 8.95 & 100.46 \\
4 & .05 & .45 & .39 & .05 & .03 & .02 & 2.63 & 8.91 & 45.84 \\
5 & .00 & .25 & .41 & .26 & .05 & .04 & 3.23 & 6.06 & 80.84 \\
6 & .01 & .10 & .34 & .37 & .09 & .09 & 3.72 & 8.00 & 65.77 \\
\hline
\end{tabular}

Note. The estimates were derived from fits by the naive mathematical model based on the theory of visual attention. $K=$ storage capacity (number of characters); $t_{0}=$ minimum effective exposure duration (in milliseconds); $C=$ processing capacity (characters per second). of 1 , eight nonsingleton distractor weights, two values of $m$, and two values of $a$ ). TVA $_{m}$ had 24 free parameters (the same parameters as $\mathrm{TVA}_{m a}$ but no values of $a$ ). Chi-square tests of the likelihood ratios, similar to the tests of the models for Experiment $1 \mathrm{~A}$, were carried out for each participant to investigate if the models were significantly different in goodness of fit. The individual participants' test values are reported in Table 12. Both models $\mathrm{TVA}_{m a}$ and $\mathrm{TVA}_{m}$ fitted the data very well; correlations between observed and predicted mean scores for the two models for each participant ranged from $r^{2}=.989$ to $r^{2}=.996$, with all $p$ values $<.001$ and $N=90$. Overall chi-square tests across participants revealed that $\mathrm{TVA}_{m a}$ did not fit the data from Experiment 2A significantly better than $\mathrm{TVA}_{m}, \chi^{2}(12)=13.89, p=$ .308. As in the analysis of Experiment $1 \mathrm{~A}$, we tested the signifi-

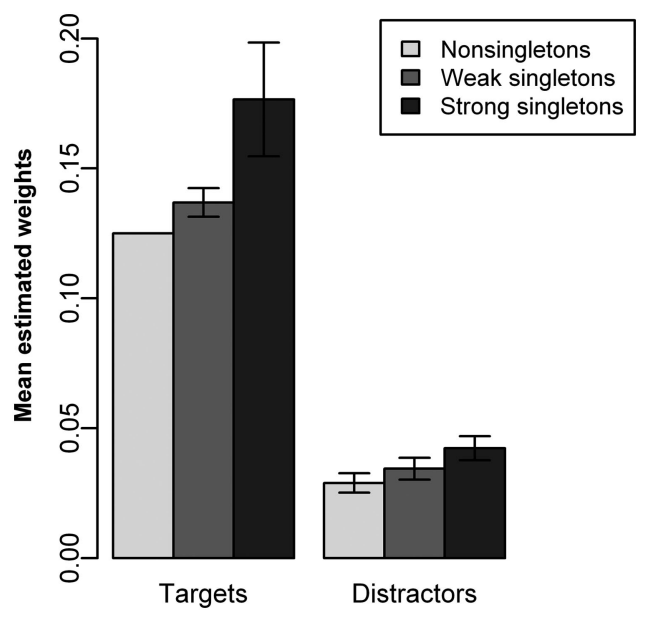

Figure 13. Group means of estimated attentional weights for each stimulus type across all spatial positions in Experiment 2A. Error bars represent standard errors of the means. The weights were normalized such that the sum of nonsingleton target weights equaled 1 for each participant. 
Targets
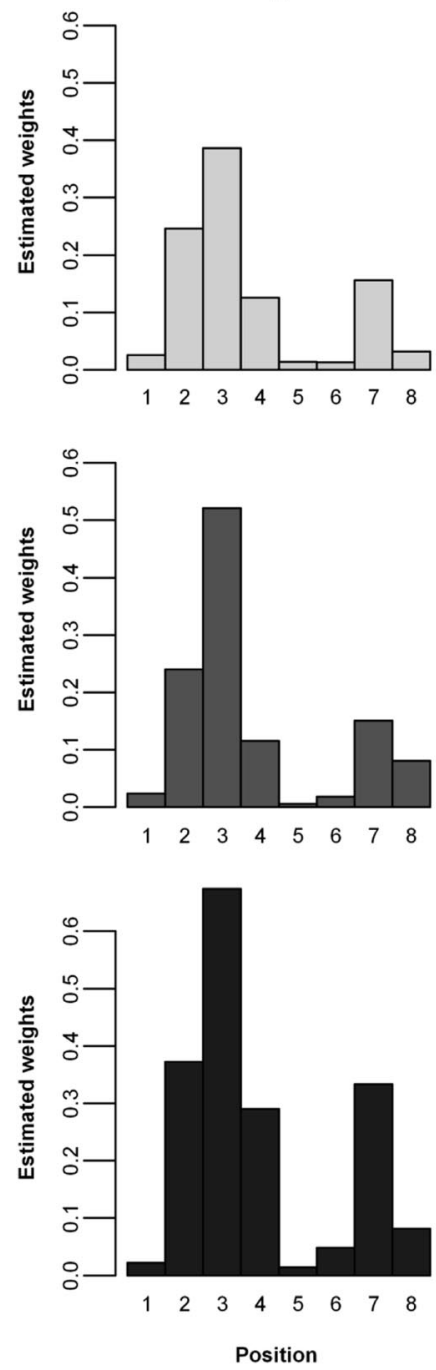

Distractors
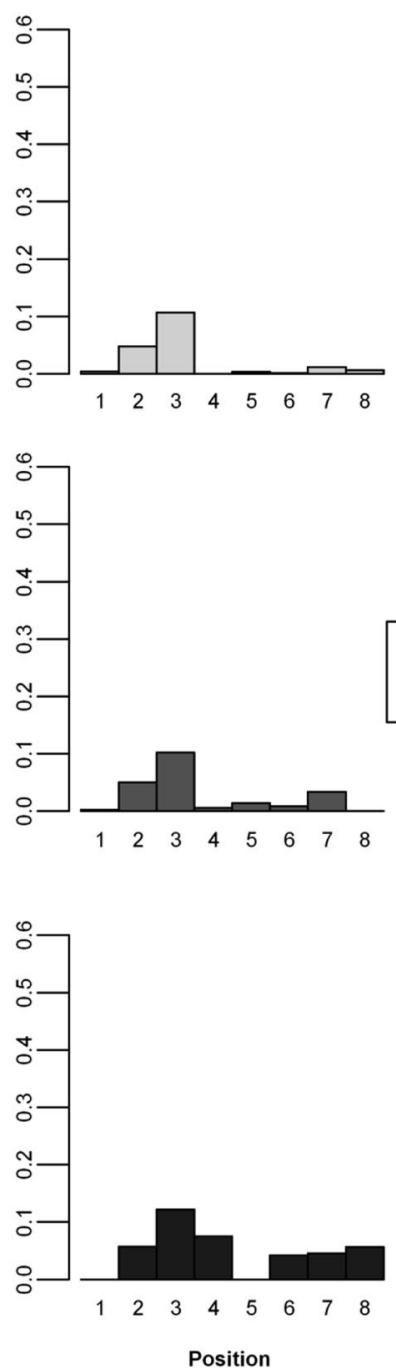

Figure 14. Estimated attentional weights for each stimulus type at each spatial position for a representative participant (Participant 1) in Experiment 2A. In terms of compass directions, Positions 1-8 represent N, NE, E, SE, S, SW, W, and NW, respectively.

cance of the multiplicative scaling of attentional weights with increase in local contrast by testing TVA $_{m}$ against the same model with the null hypothesis that $m=1\left(\mathrm{TVA}_{m \mid m=1}\right)$. The test confirmed that $\mathrm{TVA}_{m}$ fit the data significantly better than $\mathrm{TVA}_{m \mid m=1}$, $\chi^{2}(12)=305.51, p<.001$ (see Table 12 for individual statistics).

The result of the comparison between models TVA $m a$ and TVA $_{m}$ for response probabilities (the comparison reported in Table 12) agrees with the result of the comparison we made (in Table 11) between the two-parameter linear model and the one-parameter multiplicative model of the relationship between singleton and nonsingleton weights, where we found that across participants, $\chi^{2}(12)=12.78$, $p=.386$. Both comparisons support the assumption that in Experiment 2A, (a) local contrast influenced relevance-based attentional weights by multiplication of the weights by a constant that depended on the strength of the local contrast, and (b) the pertinence of contrast was zero.
The results of Experiment 2A are generally consistent with the findings from Experiment 1A. Again, both contrast and relevance influenced attentional allocation. The mean probability of correctly reporting a singleton target was significantly higher than the probability for a nonsingleton target, revealing a significant effect of contrast. However, there was also a significant effect of relevance, as evident from the significant effect of target-distractor configuration on the probability of correctly reporting a given target. The display size was constant in all three target-distractor configurations, so the effect of the configuration would be hard to explain without assuming that relevance influences initial attentional allocation. Even when the trials with the longest exposure duration (200 ms) were left out of the analysis, the effect of the targetdistractor configuration was still significant. Overall, the results of Experiment 2A confirm that both contrast and relevance influence initial attentional allocation. The attentional weights we estimated by use of the naive model corroborated this conclusion. 

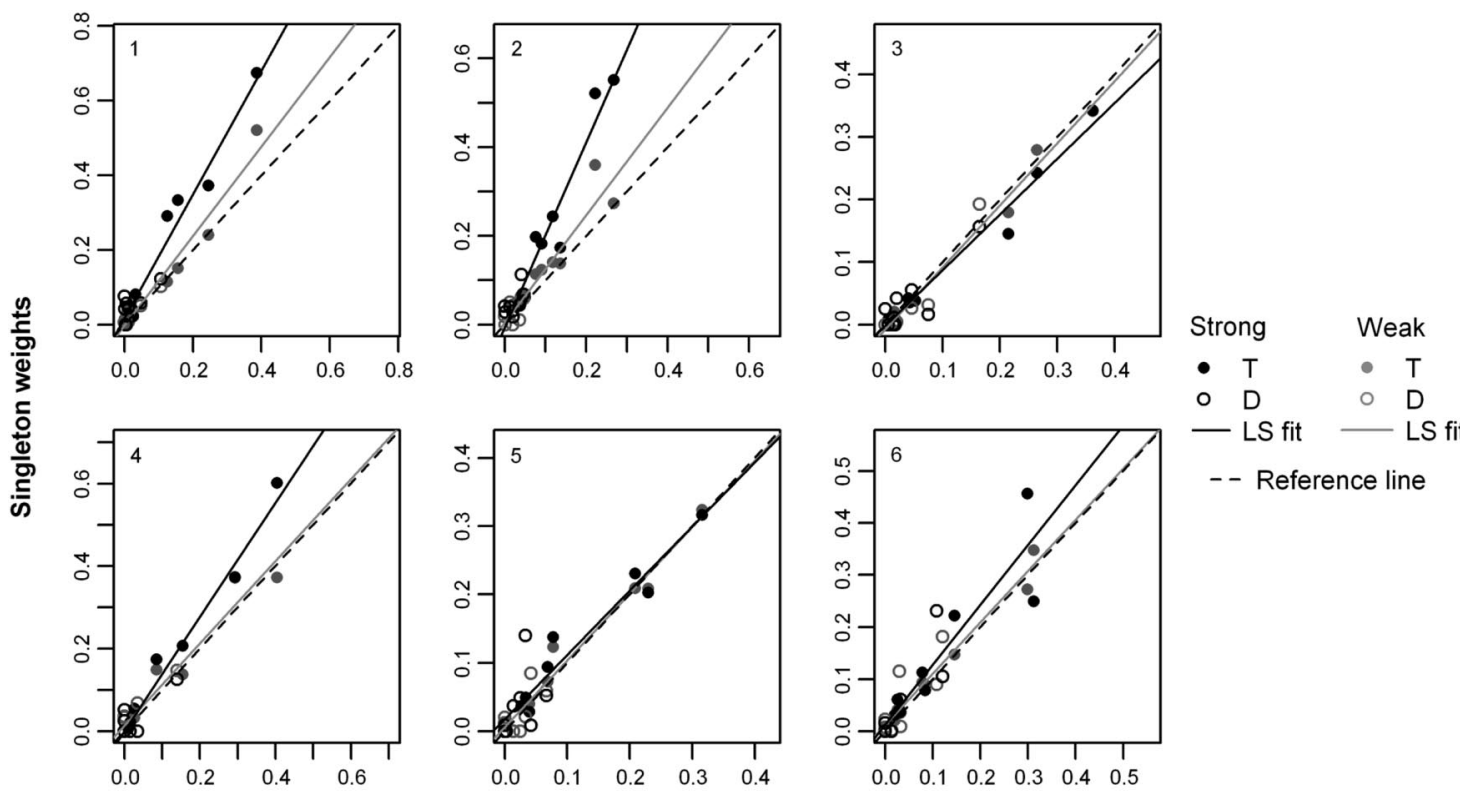

- - Reference line

Nonsingleton weights

Figure 15. Estimated attentional weights of singleton targets (T) and distractors (D) at different spatial positions plotted against the attentional weights of otherwise similar, nonsingleton targets and distractors at the same positions, for each of the six participants in Experiment 2A. For each participant, the data were fitted by two least squares (LS) straight lines, one for strong-singleton weights against nonsingleton weights and one for weak-singleton weights against nonsingleton weights. The dashed reference line is $y=x$.

The two different singleton types-strong and weak singletons-both affected the attentional weights. As expected, the strong singletons, which differed more strongly from the background color of the display and the color of the nonsingletons, had stronger effects than otherwise similar weak singletons. For both strong and weak singletons, the relationship between their weights and the weights of otherwise similar nonsingletons was reasonably well accounted for by the simple multiplicative model.

Table 11

Best Fits of Each of Three Linear Models for All Singleton Weights Against Corresponding Nonsingleton Weights for Each Participant in Experiment $2 A$

\begin{tabular}{|c|c|c|c|c|c|c|c|c|c|c|c|c|c|}
\hline \multirow[b]{3}{*}{ Participant } & \multirow[b]{3}{*}{ Singleton } & \multirow{2}{*}{\multicolumn{4}{|c|}{$\begin{array}{c}\text { Standard model } \\
\text { (free slope and intercept) }\end{array}$}} & \multicolumn{4}{|c|}{$\begin{array}{l}\text { Multiplicative model } \\
\text { (zero intercept) }\end{array}$} & \multicolumn{4}{|c|}{ Additive model (unity slope) } \\
\hline & & & & & & \multirow[b]{2}{*}{ Slope } & \multirow[b]{2}{*}{ RMSD } & \multicolumn{2}{|c|}{ LRT } & \multirow[b]{2}{*}{ Intercept } & \multirow[b]{2}{*}{ RMSD } & \multicolumn{2}{|c|}{ LRT } \\
\hline & & Intercept & Slope & $r^{2}$ & RMSD & & & $\overline{\chi^{2}(1)}$ & $p$ & & & $\chi^{2}(1)$ & $p$ \\
\hline \multirow[t]{2}{*}{1} & Weak & -0.003 & 1.20 & .955 & 0.027 & 1.19 & 0.028 & 0.12 & .725 & 0.012 & 0.035 & 7.47 & $.006^{* *}$ \\
\hline & Strong & 0.017 & 1.66 & .950 & 0.040 & 1.73 & 0.044 & 1.86 & .172 & 0.066 & 0.080 & 22.23 & $<.001^{* * * * *}$ \\
\hline \multirow[t]{2}{*}{2} & Weak & 0.005 & 1.210 & .895 & 0.031 & 1.24 & 0.031 & 0.17 & .678 & 0.020 & 0.035 & 3.65 & .056 \\
\hline & Strong & -0.006 & 2.08 & .939 & 0.040 & 2.04 & 0.040 & 0.20 & .651 & 0.074 & 0.091 & 26.25 & $<.001^{\text {****** }}$ \\
\hline \multirow[t]{2}{*}{3} & Weak & -0.008 & 0.99 & .974 & 0.017 & 0.96 & 0.018 & 1.98 & .159 & -0.008 & 0.017 & 0.03 & .862 \\
\hline & Strong & -0.002 & 0.89 & .952 & 0.021 & 0.88 & 0.021 & 0.11 & .738 & -0.011 & 0.024 & 4.12 & $.042^{*}$ \\
\hline \multirow[t]{2}{*}{4} & Weak & 0.014 & 0.99 & .944 & 0.028 & 1.05 & 0.030 & 2.50 & .114 & 0.013 & 0.028 & 0.01 & .931 \\
\hline & Strong & 0.001 & 1.38 & .960 & 0.033 & 1.38 & 0.033 & 0.01 & .935 & 0.029 & 0.055 & 16.68 & $<.001^{* * * * *}$ \\
\hline \multirow[t]{2}{*}{5} & Weak & 0.006 & 0.974 & .956 & 0.019 & 1.01 & 0.020 & 1.09 & .298 & 0.005 & 0.019 & 0.24 & .621 \\
\hline & Strong & 0.018 & 0.939 & .874 & 0.032 & 1.03 & 0.035 & 2.60 & .107 & 0.013 & 0.033 & 0.46 & .498 \\
\hline \multirow[t]{2}{*}{6} & Weak & 0.012 & 0.99 & .916 & 0.028 & 1.05 & 0.030 & 1.58 & .209 & 0.011 & 0.028 & 0.04 & .844 \\
\hline & Strong & 0.012 & 1.15 & .826 & 0.050 & 1.21 & 0.051 & 0.55 & .457 & 0.025 & 0.052 & 1.12 & .264 \\
\hline
\end{tabular}

Note. Root mean squared deviation (RMSD) is shown for comparisons between the models. The one-parameter models are tested against the two-parameter model via likelihood ratio tests (LRT); for both comparisons, the nested model differs from the standard model by one degree of freedom; $\chi^{2}$ and $p$ statistics are provided for these tests.

${ }^{*} p<.05 .{ }^{* * *} p<.01{ }^{* * * *} p<.001$. 
Table 12

Likelihood Ratio Comparisons of Fits by TVA ma $_{\text {Versus }}$ TVA $_{m}$ and $T V A_{m}$ Versus $T V A_{m \mid m=1}$, for Experiment $2 A$

\begin{tabular}{cccccc}
\hline & \multicolumn{2}{c}{ TVA $_{m a}$ vs. TVA } & & \multicolumn{2}{c}{ TVA $_{m}$ Vs. TVA } \\
\cline { 2 - 3 } Participant & & & $\chi^{2}(2)$ & $p$ \\
\hline$\chi^{2}(2)$ & 0.21 & .900 & 90.01 & $<.001^{* * * *}$ \\
1 & 3.80 & .150 & 138.54 & $<.001^{* * * *}$ \\
2 & 1.06 & .589 & 11.51 & $.003^{* * *}$ \\
3 & 3.00 & .223 & 32.29 & $<.001^{* * * *}$ \\
4 & 1.48 & .477 & 11.38 & $.003^{* * *}$ \\
5 & 4.34 & .114 & 21.78 & $<.001^{* * *}$ \\
\hline
\end{tabular}

Note. $\quad$ TVA $=$ theory of visual attention.

${ }^{* * *} p<.01{ }^{* * * *} p<.001$.

\section{Experiment 2B}

We attempted to choose the two singleton colors used in Experiment $2 \mathrm{~A}$ in such a way that the readability of stimulus characters was the same whether the local background color was the strong singleton color, the weak singleton color, or the light gray background color of the display. Experiment $2 \mathrm{~B}$ verified that this goal had been reached. The experimental procedure was generally similar to the procedure used in Experiment 1B.

\section{Method}

Participants. Six young adults, three men and three women, volunteered as participants. The participants' mean age was 30 years, with a range of 24 to 33. All participants had normal or corrected to normal eyesight, and no participants reported any history of color blindness or other visual deficiencies.

Apparatus and stimuli. The apparatus was similar to that used in Experiments 1A, 1B, and 2A. The stimulus material was similar to Experiment $2 \mathrm{~A}$ with the following exception: Only a single target and no distractors were presented. The target was randomly chosen on each trial from the same letter set as in the previous three experiments. On half the trials, the local background color of the character circle was the same as the display background color. On the other half of the trials, the local background color was equally often one or the other one of the two colors that were used as singleton colors in Experiment 2A (see Table 8). The target was shown in one of eight possible positions on an imaginary circle similar to the preceding experiment. The position of the target was randomly chosen for each trial.

Procedure. Participants were instructed to report the target as accurately as possible. When properly fixated, participants pressed enter. With a latency of $100 \mathrm{~ms}$ after the keypress, the target appeared for $10,20,30,50,70$, or $100 \mathrm{~ms}$. The target was followed by a 500-ms presentation of a masking display similar to the one used in Experiment 2A.

The participants served in two sessions of 360 trials, a total of 720 trials, yielding 40 observations per trial type (6 exposure durations $\times 3$ stimulus types). The trials were presented in random order in each session. There was as a minimum a 90-min break between sessions. Each session began with a short training session consisting of 18 trials in which participants were presented with all 18 display types, to get them attuned to the short exposure durations. After each training trial, participants received onscreen vi- sual feedback on their report (correct report, erroneous report, or no report).

\section{Results and Discussion}

The mean number of correctly reported targets was analyzed across participants for each stimulus type (gray, weak red, and strong red local background) as a function of exposure duration (see Figure 16). The mean scores were examined by a repeatedmeasures ANOVA with factors exposure duration and stimulus type. The ANOVA revealed a significant effect of exposure duration, $F(5,25)=97.34, p<.001$. There was no significant effect of stimulus type $(F<1)$, and no significant interaction, $F(10$, 50) $=1.37, p=.221$.

The three different element types, strong red, weak red, and gray, did not differ significantly from each other in the single target report. The results of the calibration experiment thus confirmed that any difference between the differently colored elements in the main experiment was not confounded by differences in readability between singleton and nonsingleton stimulus characters.

\section{General Discussion}

We set out to investigate whether both the contrast of the visual features of an object to its local surroundings (feature contrast) and the relevance of the features to the observer's goals (feature relevance) guide initial allocation of visual attention and, if so, how the two factors interact. Building on previous studies, we devised a new experimental method that seemed suited to answer our questions: a partial-report paradigm with irrelevant color singletons. Feature contrast and feature relevance were varied independently, exposures were brief and post-masked, and attentional weights were estimated by use of Bundesen's (1990) computational theory of visual attention. The results of our experiments suggest three main conclusions: (a) An object with high local feature contrast generally attracts more initial attention than an otherwise similar object with low local feature contrast, even when the feature contrast is irrelevant to the task at hand. (b) An object

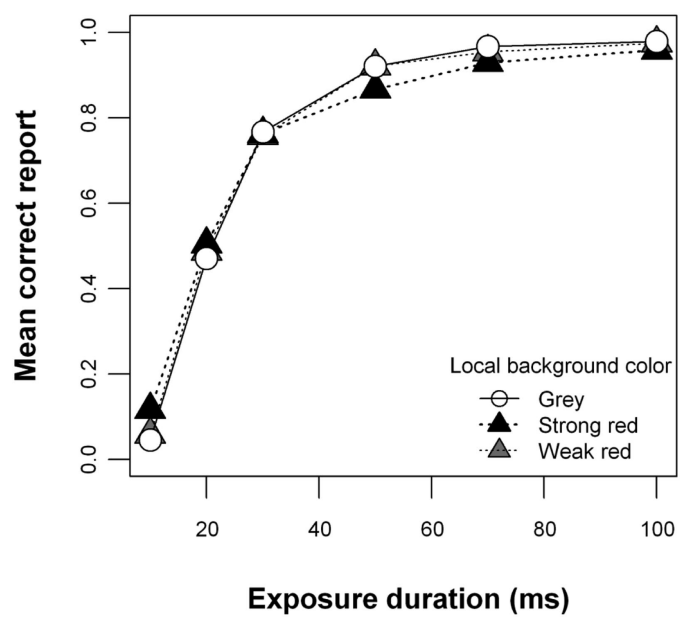

Figure 16. Group mean number of correctly reported targets for each stimulus type as a function of exposure duration in Experiment 2B. 
that is relevant to current goals generally attracts more initial attention than an object that is less relevant. (c) The attentional weight of an object is a product of a bottom-up (feature contrast) and a top-down (feature relevance) component. We elaborate these points below.

\section{Feature Contrast}

The data from our experiments revealed a distinct effect of local feature contrast on the initial allocation of attention. The probability of correctly reporting a target element was significantly higher in both Experiments $1 \mathrm{~A}$ and 2A if the target element had a high local feature contrast. In accordance with this, the estimated attentional weights were significantly higher for elements with a high contrast (color singletons) than for elements with a low contrast (nonsingletons). The main experiments (Experiments 1A and $2 \mathrm{~A}$ ) were set up in such a way that the contrast was of no relevance to the task. Furthermore, by the calibration experiments (Experiments 1B and 2B), we ensured that the high local color contrast did not influence the visibility (readability) of the target letters, so that the effect of contrast in our experiments must be considered an attentional effect.

A number of previous studies have varied static feature contrast and feature relevance independently (e.g., Folk \& Annett, 1994; Jonides \& Yantis, 1988; Theeuwes, 1990). Contrary to our findings, none of these studies has reported significant effects of task-irrelevant static contrast. We see three differences between the present experiments and previous studies, which individually or collectively may explain why our experimental method seemed more sensitive than previous methods. First, as mentioned in the introduction, the previous studies have been conducted in resource-limited conditions (speeded responses and, most often, long stimulus exposures). Our findings were obtained in data-limited conditions (unspeeded responses to brief exposures), in which performance may reflect the perceptual processing with higher fidelity (Ester \& Awh, 2008; Santee \& Egeth, 1982).

Second, the singleton in our experiments was the only feature singleton present among a relatively homogeneous set of nonsingletons. The background color was the same for all nonsingleton elements, and the shape of the outer boundary of the elements was always a circle. Thus, it seems likely that our color singleton was more salient than were the singletons in the previous studies (see, in particular, Bacon \& Egeth, 1994, Experiments 2 and 3).

Third, in a more technical vein, it can be shown that an increment from $w_{x}$ to $w_{x}+\Delta w_{x}$ in the attentional weight of an element $x$ causes a percentage increase in the relative attentional weight of $x$ (i.e., a percentage increase in $w_{x} / \sum_{z \in S} w_{z}$ ), which increases with the sum of the attentional weights of the remaining elements in the visual field (see the Appendix for a proof). Thus, by the rate equation of TVA (Equation 1), when many elements are present in the visual field and when the elements have high attentional weights, an increase in the attentional weight of a single element will have a relatively larger effect on the processing rate of that element compared to when there are few elements with low attentional weight. As an example, in the experiments of Jonides and Yantis (1988), there was never more than one target in a display (as is characteristic of resource-limited experiments) and between two and seven elements in the displays. By contrast, in our Experiments $1 \mathrm{~A}$ and $2 \mathrm{~A}$, there were between six and 10 elements in the displays and two to six of these elements were targets. For this reason, too, our experimental measures may have been more sensitive to the tested increases in feature contrast than were measures yielded by previous methods.

Many researchers have argued that objects with high local feature contrast receive attentional priority in the initial visual processing (e.g., Itti \& Koch, 2000; Theeuwes, 2010; Treisman, 1986; Wolfe, 1994). Some researchers have proposed that the initial allocation of attention is determined entirely by properties of the stimulus (e.g., Theeuwes, 2010). It is evident from our data that the probability of encoding an element was significantly increased if the element was a color singleton, even though neither the specific color feature nor the contrast signal in itself could be used to solve the task in our experiments. However, as discussed in the next section, the attentional prioritization based on contrast in our experiments did not preclude significant effects of goal relevance. Rather, we found an interaction between the contrast of an object and the relevance of that object. In our experiments, the magnitude of the effect of task-irrelevant contrast was contingent on the relevance of the object in question.

\section{Feature Relevance}

Just as local feature contrast affected allocation of attention, the data from the present study also revealed a significant effect of relevance. Two closely related findings suggested particularly strongly that relevance influences initial attentional allocation. First, in both Experiments 1A and 2A, target elements yielded significantly higher estimates of attentional weight than did distractors. Second, in Experiment 2A, the observed probabilities of correctly reporting the different target types decreased as the number of targets in the displays increased while the total number of elements in the displays was kept constant. Both results testify that elements that were relevant to the current task seized more processing resources than did less relevant elements.

The finding of an influence of relevance on initial attentional allocation is in line with many other studies (e.g., Bacon \& Egeth, 1994; Folk \& Remington, 1998; Folk et al., 1992; Leber \& Egeth, 2006; Müller, Heller, \& Ziegler, 1995; Remington, Folk, \& McLean, 2001; Wolfe et al., 1989). However, these studies have been criticized for reporting results reflecting later stages of processing rather than initial attentional allocation (Theeuwes, 2004, 2010). The above studies used reaction-time-based paradigms in which the stimuli were presented until the participants responded, typically between 400 and $900 \mathrm{~ms}$ after the stimuli came on, or in which the distracting singleton was presented $150 \mathrm{~ms}$ prior to the target stimulus display. These reaction-time-based studies leave open the interpretation that effects of relevance in these studies stem from later stages of the processing, that is, processing after the first $\sim 150 \mathrm{~ms}$ of stimulus presentation (Theeuwes, 2004, 2010). Our study responds to this criticism. As noted in the introduction, the use of brief stimulus exposures and unspeeded responses in our experiments argues against late-stage explanations of the effects of relevance.

Relevance plays a major role in TVA, as in many other theories, for instance, guided search by Wolfe (e.g., Wolfe, 1994,2006; Wolfe et al., 1989), the contingent capture theory by Folk and colleagues (e.g., Folk et al., 1992), and the dimension weighting 
account by Found, Müller, and Krummenacher (Found \& Müller, 1996; Müller \& Krummenacher, 2006). The effect of relevance in the present experiments is overall in line with these theories. However, the target category we apply is relatively complex, namely, letters, and the above theories disagree on the complexity of the categories on which visual selection can be based. Although the guided search model, the contingent capture theory, and the dimension weighting account support relevance-based attentional guidance, this guidance is thought to pertain to simple feature categories such as red, steep, and small (Wolfe, 1994; Wolfe \& Horowitz, 2004) or feature dimensions such as color, orientation, and size (Found \& Müller, 1996; Müller \& Krummenacher, 2006). Our experiments provide new evidence that alphanumeric category can guide attention, even with very brief stimulus presentations. In this way our theoretical account is similar to the attentional engagement theory of Duncan and Humphreys $(1989,1992)$ and the biased competition theory of Desimone and Duncan (1995), in which attention can be guided by more complex features. In this connection, it is interesting to note that an increasing number of studies using natural scenes and objects have found effects of task relevance in visual searches for complex categories (e.g., Alexander \& Zelinsky, 2012), even at very short exposure durations (Evans, Horowitz, \& Wolfe, 2011; Thorpe, Fize, \& Marlot, 1996). However, these effects are typically interpreted as effects of efficient feed-forward loops rather than top-down guidance by complex categories. Our results show significant top-down effects for categories of intermediate complexity. It remains for future studies to test the validity of the principles we have derived in settings with stimuli of higher complexity.

\section{Joint Effects of Contrast and Relevance}

Both feature contrast and feature relevance influence initial attentional allocation. Interestingly, we found evidence that both effects persist under the presence of the other. The probability of correctly reporting a target singleton was significantly higher than the probability of correctly reporting a target that was not a singleton, demonstrating the significant influence of contrast. At the same time, the probability of correctly reporting a target singleton was significantly influenced by the target-distractor configuration in displays of similar set size, bearing evidence that relevance and contrast concurrently influence attention. The weight estimates in Experiments 1A and 2A (see Figures 5 and 13) also confirmed the simultaneous influence of contrast and relevance. In each experiment, the estimated weights showed both significant main effects of contrast and relevance and a significant interaction between contrast and relevance.

Zehetleitner et al. (2012) have recently drawn a similar conclusion, stressing that selection is based on a continuous weighting of features and thus depends on the relative strength of top-down settings and bottom-up salience. In line with this, a number of studies have found evidence that both behavioral and electrophysiological effects of feature contrast can be modulated by top-down settings (Kim \& Cave, 1999; Leber \& Egeth, 2006; Müller et al., 2009; Töllner, Müller, \& Zehetleitner, 2012; Töllner, Zehetleitner, Gramann, \& Müller, 2010; Zehetleitner, Krummenacher, Geyer, Hegenloh, \& Müller, 2011).

\section{Models of Singleton Weights}

As shown in the TVA-Based Predictions section, traditional TVA suggests an additive model of singleton weights: By the traditional weight equation (Equation 2), an increment in $\eta(x$, contrast) by a certain amount, $\Delta \eta(x$, contrast $)$, yields an increment by $\Delta \eta(x$, contrast $) \pi_{\text {contrast }}$ in $w_{x}$, regardless of the prior size of $w_{x}$. Thus, other things equal, changing the status of an object $x$ from nonsingleton to singleton increases the attentional weight of the object by adding an increment, which is independent of the prior size of $w_{x}$.

We tested the additive model of singleton weights for each type of singleton (one type in Experiment 1A, two types-strong and weak-in Experiment 2A) by first estimating attentional weights of singleton targets (two types in Experiment 2A), nonsingleton targets, singleton distractors (two types in Experiment 2A), and nonsingleton distractors at all possible locations in our stimulus displays by fitting the data by TVA with the attentional weights as free parameters (the naive model). We then plotted the estimated weights of singleton objects against the estimated weights of otherwise similar nonsingleton objects. According to the additive model of singleton weights, the plotted points should lie on a straight line with a slope of 1 and an intercept of $\Delta \eta(x$, contrast $)$ $\pi_{\text {contrast }}$. Actually, the plotted points lay approximately on a straight line, but the fit obtained by a linear model with two free parameters (intercept and slope) was significantly better than the fit by the additive model of singleton weights in which the intercept was the only free parameter $(p<.001$ for both Experiment $1 \mathrm{~A}$ and Experiment 2A), so the additive model was inferior to the two-parameter linear model of singleton weights.

\section{Contrast and Relevance in Computation of Attentional Weights}

In the Discussion of Experiments $1 \mathrm{~A}$ and $1 \mathrm{~B}$ section, we explained the two-parameter linear model of singleton weights by proposing a generalization (Equation 3) of the weight equation of TVA, the generalization

$$
w_{x}=\kappa_{x} \sum_{j \in R} \eta(x, j) \pi_{j},
$$

and deriving the two-parameter linear model of singleton weights from this generalized weight equation. In the generalized weight equation - the COntrast-RElevance (CORE) equation- $\kappa_{x}$ is the strength of the local feature contrast of object $x$, and the summation across $j$ subsumes $\eta(x$, contrast $) \pi_{\text {contrast }}$. The CORE equation reduces to the traditional weight equation when the local feature contrast, $\mathrm{\kappa}_{x}$, is the same for every object $x$. Without loss of generality, $\kappa_{\text {nonsingleton }}$ can be set at a value of 1 . In this case, the slope of the two-parameter linear model of singleton weights equals $\kappa_{\text {singleton }}$, whereas the intercept equals the product of $\kappa_{\text {singleton }}$, the increment $\eta$ (singleton, contrast) $-\eta$ (nonsingleton, contrast), and $\pi_{\text {contrast }}$ (see Equations $4-6$ ). Thus, the intercept should be positive if $\pi_{\text {contrast }}>0$. If $\pi_{\text {contrast }}=0$, the intercept should also be 0 . In this special case, the two-parameter linear model of singleton weights reduces to a one-parameter multiplicative model, in which the weight of a singleton object is directly proportional to the weight of an otherwise similar nonsingleton object, $\kappa_{\text {singleton }}$ being the constant of proportionality. 
The results of Experiments 1A and 2A were well accounted for by models based on TVA with the CORE equation. Fits to the response probabilities based on the assumption that $\pi_{\text {contrast }}=0$ were pitted against fits based on the more general assumption that $\pi_{\text {contrast }} \geq 0$. For Experiment $1 \mathrm{~A}$, fits by TVA with the CORE equation and the assumption that $\pi_{\text {contrast }} \geq 0$ were not significantly better than fits by TVA with the CORE equation and the assumption that $\pi_{\text {contrast }}=0$ (see Table 7), suggesting that in Experiment $1 \mathrm{~A}, \pi_{\text {contrast }}$ was close to 0 . However, across participants in Experiment 1A, the relationship between the singleton versus nonsingleton weights estimated by the naive model was significantly better fitted by the two-parameter linear model compared with the one-parameter multiplicative model (see Table 6), suggesting that $\pi_{\text {contrast }}>0$. Presumably, $\pi_{\text {contrast }}$ was positive for one or more participants in Experiment $1 \mathrm{~A}$ but was not much greater than 0 .

As was the case for Experiment 1A, fits to the response probabilities in Experiment 2A by TVA with the CORE equation and the assumption that $\pi_{\text {contrast }} \geq 0$ were not significantly better than fits by TVA with the CORE equation and the assumption that $\pi_{\text {contrast }}=0$ (see Table 12), suggesting that in Experiment 2A, $\pi_{\text {contrast }}$ was close to 0 . Furthermore, compared with the oneparameter multiplicative model for singleton weights, the twoparameter linear model for singleton weights did not provide significantly better fits to the plots of singleton versus nonsingleton weights estimated by the naive model (see Table 11, columns 9 and 10 ), again suggesting that $\pi_{\text {contrast }} \approx 0$. Presumably, $\pi_{\text {contrast }}$ was close to 0 for the participants in Experiment $2 \mathrm{~A}$.

Whereas the results lend support to TVA with the CORE equation, the implications concerning the pertinence of local feature contrast are less certain. We speculate that in very many situations, paying attention to singletons by letting $\pi_{\text {contrast }}>0$ is sound from a biological point of view. Thus, $\pi_{\text {contrast }}$ may be positive by default. In special cases we may know that contrast is task irrelevant. In such cases, performance may be optimized by setting $\pi_{\text {contrast }}=0$ so that attentional weights reflect the requirements of the task with maximum fidelity. In Experiments $1 \mathrm{~A}$ and $2 \mathrm{~A}$, contrast in color was known to be task irrelevant, so in both experiments, participants may have attempted to set $\pi_{\text {contrast }}$ close to 0 .

The $\kappa$ component presumably reflects the contrast between the physical properties of the stimulus and its surroundings, akin to the activation values computed in the saliency map models (e.g., Itti \& Koch, 2000; Koch \& Ullman, 1985), the feature dissimilarities described by Nothdurft (e.g., 1992, 1993), and the bottom-up activations of Wolfe (1994). We speculate that the weight of the singleton elements in the additional singleton experiments may be influenced both by the $\kappa$ component and by a positive pertinence of contrast, as contrast has a certain relevance to the task in these experiments. Such a concurrent effect of $\kappa$ and positive pertinence of contrast should increase the overall effect of contrast, in line with the results reported in the previous literature (e.g., Theeuwes, 1991, 1992).

The multiplicative nature of the CORE weight equation implies that the effect of the contrast of an object depends on the relevance of the object, and vice versa. If the relevance (i.e., the sum of $\eta(x$, i) $\pi_{i}$ products) of an object approaches zero, then so does the total attentional weight of that object, regardless of the contrast of the object. Similarly, when the contrast approaches zero, so does the weight, regardless of the relevance.

Whereas the CORE weight equation is derived from experiments with relatively simple stimuli, the principles should generalize to visual perception of complex stimuli, such as natural scenes: The contribution from the feature contrast component should be interdependent with the contribution from the feature relevance component, and this interdependence should be well described by a multiplicative relation. Although the components of feature contrast and of feature relevance are harder to isolate in a natural scene, it remains an important task for future studies to confirm the generality of the CORE equation.

\section{Other Models Relating Contrast and Relevance}

Other models of visual attention have proposed that initial selection depends on both a contrast- and a relevance-based component (e.g., Müller \& Krummenacher, 2006; Navalpakkam \& Itti, 2002; Wolfe, 1994), and recent studies have suggested that selection depends on the relative strength of the two components (Zehetleitner et al., 2012). In the guided search model, Wolfe et al. (1989) introduced the idea that attentional selection is based on activations in an overall activation map that is computed by adding relevance-based, top-down activations and contrast-based bottom-up activations. In a way, our introduction of $\kappa$ in the weight equation of TVA makes TVA more similar to guided search and related models such as the dimension weighting account (Found \& Müller, 1996). However, in guided search, contrast and relevance activations are added together, whereas, in TVA with the CORE equation, the attentional weight of an object is a product of the contrast and the relevance of the object.

As noted, computation of $\kappa$ values may resemble the saliency map activations described by Ullman, Koch, and Itti, among others (Itti \& Koch, 2000; Koch \& Ullman, 1985). Interestingly, Navalpakkam and Itti (2002) have proposed a model of relevance- and contrast-based attention guidance, building on the earlier models of saliency maps. In the model of Navalpakkam and Itti (2002), the attention guidance map consists of pointwise products of salience and task relevance, similar to the products of contrast and relevance proposed in the CORE equation. The data from the present experiments and the equation we have derived from these data are thus in line with trends in the literature on computer vision. Note, however, that our $\kappa$ parameter pertains to objects, whereas the above-mentioned models operate on a pixel-based level. Recent developments in computer vision suggest that object-based computation of contrast may be more efficient and may better reflect qualitative aspects of human attention than does the pixel-based approach (Aziz \& Mertsching, 2008; Wischnewski, Belardinelli, Schneider, \& Steil, 2010). Our introduction of the $\kappa$ component is in line with these new advances.

\section{Concluding Remarks}

We have attempted to settle the long debate on bottom-up capture versus top-down control of visual attention by experimental analysis and computational modeling. Our studies seem to show that both local feature contrast and relevance to the observer's goals affect initial allocation of attention. In particular, the results suggest a generalization of the weight equation of TVA 
(Bundesen, 1990) such that the attentional weight of an object equals a product of a bottom-up, local feature contrast component and a top-down, feature relevance component.

\section{References}

Alexander, R. G., \& Zelinsky, G. J. (2012). Effects of part-based similarity on visual search: The Frankenbear experiment. Vision Research, 54, 20-30. doi:10.1016/j.visres.2011.12.004

Aziz, M. Z., \& Mertsching, B. (2008). Fast and robust generation of feature maps for region-based visual attention. IEEE Transactions on Image Processing, 17, 633-644. doi:10.1109/TIP.2008.919365

Bacon, W. F., \& Egeth, H. E. (1994). Overriding stimulus-driven attentional capture. Perception \& Psychophysics, 55, 485-496.

Belopolsky, A. V., \& Theeuwes, J. (2010). No capture outside the attentional window. Vision Research, 50, 2543-2550. doi:10.1016/j.visres .2010 .08 .023

Belopolsky, A. V., Zwaan, L., Theeuwes, J., \& Kramer, A. F. (2007). The size of an attentional window modulates attentional capture by color singletons. Psychonomic Bulletin \& Review, 14, 934-938. doi:10.3758/ BF03194124

Bundesen, C. (1990). A theory of visual attention. Psychological Review, 97, 523-547. doi:10.1037/0033-295X.97.4.523

Bundesen, C. (1998). A computational theory of visual attention. Philosophical Transactions of the Royal Society of London, Series B: Biological Sciences, 353, 1271-1281. doi:10.1098/rstb.1998.0282

Bundesen, C., \& Habekost, T. (2008). Principles of visual attention: Linking mind and brain. New York, NY: Oxford University Press.

Bundesen, C., Habekost, T., \& Kyllingsbæk, S. (2005). A neural theory of visual attention: Bridging cognition and neurophysiology. Psychological Review, 112, 291-328. doi:10.1037/0033-295X.112.2.291

Bundesen, C., Habekost, T., \& Kyllingsbæk, S. (2011). A neural theory of visual attention and short-term memory (NTVA). Neuropsychologia, 49, 1446-1457. doi:10.1016/j.neuropsychologia.2010.12.006

Desimone, R., \& Duncan, J. (1995). Neural mechanisms of selective visual attention. Annual Review of Neuroscience, 18, 193-222. doi:10.1146/ annurev.ne.18.030195.001205

Duncan, J., Bundesen, C., Olson, A., Humphreys, G., Chavda, S., \& Shibuya, H. (1999). Systematic analysis of deficits in visual attention. Journal of Experimental Psychology: General, 128, 450-478. doi: 10.1037/0096-3445.128.4.450

Duncan, J., \& Humphreys, G. W. (1989). Visual search and stimulus similarity. Psychological Review, 96, 433-458. doi:10.1037/0033-295X .96 .3 .433

Duncan, J., \& Humphreys, G. W. (1992). Beyond the search surface: Visual search and attentional engagement. Journal of Experimental Psychology: Human Perception and Performance, 18, 578-588. doi: 10.1037/0096-1523.18.2.578

Dyrholm, M., Kyllingsbæk, S., Espeseth, T., \& Bundesen, C. (2011). Generalizing parametric models by introducing trial-by-trial parameter variability: The case of TVA. Journal of Mathematical Psychology, 55, 416-429. doi:10.1016/j.jmp.2011.08.005

Ester, E. F., \& Awh, E. (2008). The processing locus of interference from salient singleton distractors. Visual Cognition, 16, 166-181. doi: 10.1080/13506280701489510

Evans, K. K., Horowitz, T. S., \& Wolfe, J. M. (2011). When categories collide: Accumulation of information about multiple categories in rapid scene perception. Psychological Science, 22, 739-746. doi:10.1177/ 0956797611407930

Folk, C. L., \& Annett, S. (1994). Do locally defined feature discontinuities capture attention? Perception \& Psychophysics, 56, 277-287.

Folk, C. L., \& Remington, R. W. (1998). Selectivity in distraction by irrelevant featural singletons: Evidence for two forms of attentional capture. Journal of Experimental Psychology: Human Perception and Performance, 24, 847-858. doi:10.1037/0096-1523.24.3.847

Folk, C. L., \& Remington, R. W. (2010). A critical evaluation of the disengagement hypothesis. Acta Psychologica, 135, 103-105. doi: 10.1016/j.actpsy.2010.04.012

Folk, C. L., Remington, R. W., \& Johnston, J. C. (1992). Involuntary covert orienting is contingent on attentional control settings. Journal of Experimental Psychology: Human Perception and Performance, 18, 1030-1044. doi:10.1037/0096-1523.18.4.1030

Folk, C. L., Remington, R. W., \& Wright, J. H. (1994). The structure of attentional control: Contingent attentional capture by apparent motion, abrupt onset, and color. Journal of Experimental Psychology: Human Perception and Performance, 20, 317-329. doi:10.1037/0096-1523.20 .2 .317

Found, A., \& Müller, H. J. (1996). Searching for unknown feature targets on more than one dimension: Investigating a "dimension-weighting" account. Perception \& Psychophysics, 58, 88-101.

Habekost, T., \& Starrfelt, R. (2009). Visual attention capacity: A review of TVA-based patient studies. Scandinavian Journal of Psychology, 50, 23-32. doi:10.1111/j.1467-9450.2008.00681.x

Itti, L., \& Koch, C. (2000). A saliency-based search mechanism for overt and covert shifts of visual attention. Vision Research, 40, 1489-1506. doi:10.1016/S0042-6989(99)00163-7

Jonides, J., \& Yantis, S. (1988). Uniqueness of abrupt visual onset in capturing attention. Perception \& Psychophysics, 43, 346-354.

Kim, M.-S., \& Cave, K. R. (1999). Top-down and bottom-up attentional control: On the nature of interference from a salient distractor. Perception \& Psychophysics, 61, 1009-1023. doi:10.3758/BF03207609

Koch, C., \& Ullman, S. (1985). Shifts in selective visual attention: Towards the underlying neural circuitry. Human Neurobiology, 4, 219-227.

Kyllingsbæk, S. (2006). Modeling visual attention. Behavior Research Methods, 38, 123-133. doi:10.3758/BF03192757

Leber, A. B., \& Egeth, H. E. (2006). It's under control: Top-down search strategies can override attentional capture. Psychonomic Bulletin \& Review, 13, 132-138. doi:10.3758/BF03193824

Logan, G. D. (1996). The CODE theory of visual attention: An integration of space-based and object-based attention. Psychological Review, 103, 603-649. doi:10.1037/0033-295X.103.4.603

Logan, G. D. (2002). An instance theory of attention and memory. Psychological Review, 109, 376-400. doi:10.1037/0033-295X.109.2.376

Logan, G. D., \& Gordon, R. D. (2001). Executive control of visual attention in dual-task situations. Psychological Review, 108, 393-434. doi:10.1037/0033-295X.108.2.393

Müller, H. J., Geyer, T., Zehetleitner, M., \& Krummenacher, J. (2009). Attentional capture by salient color singleton distractors is modulated by top-down dimensional set. Journal of Experimental Psychology: Human Perception and Performance, 35, 1-16. doi:10.1037/0096-1523.35.1.1

Müller, H. J., Heller, D., \& Ziegler, J. (1995). Visual search for singleton feature targets within and across feature dimensions. Perception \& Psychophysics, 57, 1-7.

Müller, H. J., \& Krummenacher, J. (2006). Locus of dimension weighting Preattentive or postselective? Visual Cognition, 14, 490-513.

Müller, H. J., Töllner, T., Zehetleitner, M., Geyer, T., Rangelov, D., \& Krummenacher, J. (2010). Dimension-based attention modulates feedforward visual processing. Acta Psychologica, 135, 117-122. doi: 10.1016/j.actpsy.2010.05.004

Navalpakkam, V., \& Itti, L. (2002). A goal oriented attention guidance model. Lecture Notes in Computer Science, 2525, 81-118. doi:10.1007/ 3-540-36181-2_45

Nothdurft, H.-C. (1992). Feature analysis and the role of similarity in preattentive vision. Perception \& Psychophysics, 52, 355-375.

Nothdurft, H.-C. (1993). Saliency effects across dimensions in visual search. Vision Research, 33, 839-844. doi:10.1016/00426989(93)90202-8 
Remington, R. W., Folk, C. L., \& McLean, J. P. (2001). Contingent attentional capture or delayed allocation of attention? Perception \& Psychophysics, 63, 298-307.

Santee, J. L., \& Egeth, H. E. (1982). Do reaction time and accuracy measure the same aspects of letter recognition? Journal of Experimental Psychology: Human Perception and Performance, 8, 489-501. doi: 10.1037/0096-1523.8.4.489

Shibuya, H., \& Bundesen, C. (1988). Visual selection from multielement displays: Measuring and modeling effects of exposure duration. Journal of Experimental Psychology: Human Perception and Performance, 14, 591-600. doi:10.1037/0096-1523.14.4.591

Theeuwes, J. (1990). Perceptual selectivity is task-dependent: Evidence from selective search. Acta Psychologica, 74, 81-89. doi:10.1016/00016918(90)90036-F

Theeuwes, J. (1991). Cross-dimensional perceptual selectivity. Perception \& Psychophysics, 50, 184-193.

Theeuwes, J. (1992). Perceptual selectivity for color and form. Perception \& Psychophysics, 51, 599-606.

Theeuwes, J. (2004). Top-down search strategies cannot override attentional capture. Psychonomic Bulletin \& Review, 11, 65-70. doi:10.3758/ BF03206462

Theeuwes, J. (2010). Top-down and bottom-up control of visual selection. Acta Psychologica, 135, 77-99. doi:10.1016/j.actpsy.2010.02.006

Theeuwes, J., \& Burger, R. (1998). Attentional control during visual search: The effect of irrelevant singletons. Journal of Experimental Psychology: Human Perception and Performance, 24, 1342-1353. doi: 10.1037/0096-1523.24.5.1342

Thorpe, S., Fize, D., \& Marlot, C. (1996, June 6). Speed of processing in the human visual system. Nature, 381, 520-522.

Töllner, T., Müller, H. J., \& Zehetleitner, M. (2012). Top-down dimensional weight set determines the capture of visual attention: Evidence from the PCN component. Cerebral Cortex, 22, 1554-1563. doi: 10.1093/cercor/bhr231

Töllner, T., Zehetleitner, M., Gramann, K., \& Müller, H. J. (2010). Topdown weighting of visual dimensions: Behavioral and electrophysiolog- ical evidence. Vision Research, 50, 1372-1381. doi:10.1016/j.visres .2009.11.009

Töllner, T., Zehetleitner, M., Gramann, K., \& Müller, H. J. (2011). Stimulus saliency modulates pre-attentive processing speed in human visual cortex. PLOS ONE, 6(1), e16276. doi:10.1371/journal.pone.0016276

Treisman, A. (1986). Features and objects in visual processing. Scientific American, 255(5), 114-125. doi:10.1038/scientificamerican1186-114B

Wischnewski, M., Belardinelli, A., Schneider, W. X., \& Steil, J. J. (2010). Where to look next? Combining static and dynamic proto-objects in a TVA-based model of visual attention. Journal of Cognitive Computation, 2, 326-343. doi:10.1007/s12559-010-9080-1

Wolfe, J. M. (1994). Guided Search 2.0: A revised model of visual search. Psychonomic Bulletin \& Review, 1, 202-238. doi:10.3758/BF03200774

Wolfe, J. M. (2006). Guided Search 4.0: Current progress with a model of visual search. In W. Grey (Ed.), Integrated models of cognitive systems (pp. 99-119). New York, NY: Oxford University Press.

Wolfe, J. M., Cave, K. R., \& Franzel, S. L. (1989). Guided search: An alternative to the feature integration model for visual search. Journal of Experimental Psychology: Human Perception and Performance, 15, 419-433. doi:10.1037/0096-1523.15.3.419

Wolfe, J. M., \& Horowitz, T. S. (2004). What attributes guide the deployment of visual attention and how do they do it? Nature Reviews Neuroscience, 5, 495-501. doi:10.1038/nrn1411

Yantis, S., \& Egeth, H. E. (1999). On the distinction between visual salience and stimulus-driven attentional capture. Journal of Experimental Psychology: Human Perception and Performance, 25, 661-676. doi:10.1037/0096-1523.25.3.661

Zehetleitner, M., Goschy, H., \& Müller, H. J. (2012). Top-down control of attention: It's gradual, practice-dependent, and hierarchically organized. Journal of Experimental Psychology: Human Perception and Performance. Advance online publication. doi:10.1037/a0027629

Zehetleitner, M., Krummenacher, J., Geyer, J., Hegenloh, M., \& Müller, H. J. (2011). Dimension intertrial and cuing effects in localization: Support for pre-attentively weighted one-route models of saliency. Attention, Perception, \& Psychophysics, 73, 349-363. doi:10.3758/ s13414-010-0035-0 


\section{Appendix}

\section{Effect on Processing Speed Caused by an Increment in Attentional Weight}

Consider the effect of incrementing the attentional weight of element $x$ from a value of $w_{x}$ to a value of $w_{x}+\Delta w_{x}$, where $w_{x}>$ 0 and $\Delta w_{x}>0$. Let $w_{\text {other }}$ be the sum of the attentional weights of all other elements in the visual field. The increment in the attentional weight of element $x$ causes an increase in the relative attentional weight of element $x$ from a value of

$$
\frac{w_{x}}{w_{x}+w_{\text {other }}}
$$

to a value of

$$
\frac{w_{x}+\Delta w_{x}}{w_{x}+\Delta w_{x}+w_{\text {other }}}
$$

corresponding to a multiplication by the factor

$$
\frac{\left(w_{x}+\Delta w_{x}\right)\left(w_{x}+w_{\text {other }}\right)}{\left(w_{x}+\Delta w_{x}+w_{\text {other }}\right) w_{x}}
$$

or a percentage increase of

$$
f\left(w_{x}, \Delta w_{x}, w_{\text {other }}\right)=\left[\frac{\left(w_{x}+\Delta w_{x}\right)\left(w_{x}+w_{\text {other }}\right)}{\left(\mathrm{w}_{\mathrm{x}}+\Delta w_{x}+w_{\text {other }}\right) w_{x}}-1\right] \times 100 \%
$$

We shall prove that the percentage increase, $f\left(w_{x}, \Delta w_{x}, w_{\text {other }}\right)$, in the relative attentional weight of $x$, caused by the increment from $w_{x}$ to $w_{x}+\Delta w_{x}$ in the attentional weight of element $x$, is a monotonically increasing function of the sum of the attentional weights of all other elements in the visual field, $w_{\text {other }}$. Clearly, for given values of $w_{x}$ and $\Delta w_{x}, f\left(w_{x}, \Delta w_{x}, w_{\text {other }}\right)$ is a function of $w_{\text {other }}$. If we apply the rule for the derivative of the quotient of two differentiable functions and reduce the result, the derivative of the quotient in Equation A1 with respect to $w_{\text {other }}$ reduces to

$$
\frac{\left(w_{x}+\Delta w_{x}\right) w_{x} \Delta w_{x}}{\left(w_{x}+\Delta w_{x}+w_{\text {other }}\right)^{2} w_{x}^{2}}
$$

which is positive because $w_{x}>0$ and $\Delta w_{x}>0$. Accordingly, for any given values of $w_{x}$ and $\Delta w_{x}, f\left(w_{x}, \Delta w_{x}, w_{\text {other }}\right)$ is a monotonically increasing function of $w_{\text {other }}$, which was to be proved. By the rate equation of TVA (Equation 1), the proof implies that the percentage increase in the speed of encoding categorizations of $x$ into VSTM, caused by the increment from $w_{x}$ to $w_{x}+\Delta w_{x}$ in the attentional weight of element $x$, is a monotonically increasing function of the sum of the attentional weights of all other elements in the visual field, $w_{\text {other }}$.

Received February 16, 2012 Revision received July 4, 2012 Accepted July 9, 2012 